\title{
Periastron advance in spinning black hole binaries: Gravitational self-force from numerical relativity
}

\author{
Alexandre Le Tiec, ${ }^{1}$ Alessandra Buonanno, ${ }^{1}$ Abdul H. Mroué, ${ }^{2}$ Harald P. Pfeiffer, ${ }^{2,3}$ Daniel A. Hemberger, ${ }^{4,5}$ \\ Geoffrey Lovelace, ${ }^{6,4}$ Lawrence E. Kidder, ${ }^{5}$ Mark A. Scheel, ${ }^{4}$ Bela Szilágyi, ${ }^{4}$ Nicholas W. Taylor, ${ }^{4}$ and Saul A. Teukolsky ${ }^{5}$ \\ ${ }^{1}$ Department of Physics, Maryland Center for Fundamental Physics \& Joint Space-Science Institute, University of Maryland, \\ College Park, Maryland 20742, USA \\ ${ }^{2}$ Canadian Institute for Theoretical Astrophysics, University of Toronto, Toronto, Ontario M5S 3H8, Canada \\ ${ }^{3}$ Canadian Institute for Advanced Research, 180 Dundas Street West, Toronto, Ontario M5G 1Z8, Canada \\ ${ }^{4}$ Theoretical Astrophysics 350-17, California Institute of Technology, Pasadena, California 91125, USA \\ ${ }^{5}$ Center for Radiophysics and Space Research, Cornell University, Ithaca, New York 14853, USA \\ ${ }^{6}$ Gravitational Wave Physics and Astronomy Center, California State University Fullerton, Fullerton, California 92831, USA
}

(Received 2 September 2013; published 9 December 2013)

\begin{abstract}
We study the general relativistic periastron advance in spinning black hole binaries on quasicircular orbits, with spins aligned or antialigned with the orbital angular momentum, using numerical-relativity simulations, the post-Newtonian approximation, and black hole perturbation theory. By imposing a symmetry by exchange of the bodies' labels, we devise an improved version of the perturbative result and use it as the leading term of a new type of expansion in powers of the symmetric mass ratio. This allows us to measure, for the first time, the gravitational self-force effect on the periastron advance of a nonspinning particle orbiting a Kerr black hole of mass $M$ and spin $S=-0.5 M^{2}$, down to separations of order $9 M$. Comparing the predictions of our improved perturbative expansion with the exact results from numerical simulations of equal-mass and equal-spin binaries, we find a remarkable agreement over a wide range of spins and orbital separations.
\end{abstract}

DOI: 10.1103/PhysRevD.88.124027

\section{INTRODUCTION}

Accounting for the observed anomalous advance of Mercury's perihelion was the first successful test of Einstein's theory of general relativity [1]. More recently, the same effect—but with a much larger amplitude, of the order a few degrees per year-has been observed in the orbital motion of binary pulsars [2,3]. Today, the prospect of observing gravitational radiation from binary systems of compact objects (black holes and neutron stars) is triggering further interest in the relativistic periastron advance. A worldwide effort is currently underway to achieve the first direct detection of gravitational waves by using kilometer-scale, ground-based laser interferometers such as advanced LIGO [4] and advanced Virgo [5], as well as future space-based antennas, such as the eLISA mission [6]. The detection and analysis of these signals require very accurate theoretical predictions, for use as template waveforms to be cross correlated against the output of the detectors. Hence, an accurate modeling of the relativistic orbital dynamics of compact-object binary systems is crucially needed.

For binaries with small orbital velocities/large separations, but otherwise arbitrary mass ratios, the periastron advance has been computed to increasingly high orders using the post-Newtonian (PN) approximation to general relativity [7]. For nonspinning binaries moving on generic (bound) orbits, the 1PN, 2PN, and 3PN results were derived in Refs. [8-10]. Spin-orbit and spin-spin effects were computed up to $3.5 \mathrm{PN}$ order for aligned or antialigned
PACS numbers: 04.25.D-, 04.25.dg, 04.25.Nx, 04.30.-w

spins [11,12], as well as for generic spin orientations in special binary configurations [13,14]; see Ref. [9] for earlier references. For binaries with extreme mass ratios, the orbital motion can be studied using black-hole perturbation theory [15-17]. In the test-mass approximation, the periastron advance of a nonspinning particle on a generic (bound) geodesic orbit around a Schwarzschild or Kerr black hole has been computed in Refs. [18,19]. The corrections linear and quadratic in the spin of the small body were computed in the companion paper [20], for nearly circular orbits. The first-order mass-ratio correction to the geodesic result was obtained in Ref. [21] for a Schwarzschild background, but the result is still unknown in the Kerr case. Using the effective-one-body (EOB) formalism [22-25], the periastron advance has been computed for nonspinning [26] as well as for spinning compact binaries [20] on quasicircular orbits.

Following the breakthrough in the numerical simulation of the late inspiral and merger of binary black hole (BBH) systems [27-29] (see Ref. [30] for a recent review), it has recently become possible to study the periastron advance using fully nonlinear numerical relativity (NR) simulations. The first NR results for the periastron advance were presented in Ref. [31], and an improved analysis using longer and more accurate numerical simulations was done in Ref. [32]. More recently, the periastron advance has also been measured in a mixed neutron star/ black hole binary [33]. In this paper we extend the earlier works [31,32] for nonspinning black hole binaries to 
spinning systems. We make use of accurate NR simulations of the late inspiral of spinning BBHs on quasicircular orbits, with spins aligned or antialigned with the orbital angular momentum. The simulations we analyze have two different origins: (i) the series of equal-mass, equal-spin binaries presented in Refs. [34,35], with a focus on the properties of binaries with nearly extremal spins, and (ii) the unequal-mass spinning simulations presented in Ref. [36].

After deriving explicit expressions for the periastron advance at the highest PN order currently known, we compare those predictions to the NR data. We then use the mathematical structure of the PN expansion for the periastron advance, together with explicit formulas for the periastron advance of a nonspinning and spinning particle in Kerr spacetime, to derive an improved version of the perturbative result that is fully symmetrized by exchange of the bodies' labels. Indeed, earlier works [32,37-42] suggested that working with a "symmetrized background" can successfully extend the domain of validity of perturbative calculations. Finally, we show how to employ the improved, perturbative result to extract the gravitational self-force (GSF) correction to the periastron advance from NR simulations. As a proof of principle, we first use the NR simulations of nonspinning $\mathrm{BBH}$ systems with mass ratios $1-8$, extract the GSF correction to the periastron advance and compare it with the known, exact result from perturbative calculations [21]. Then, we consider NR simulations of single-spin BBH systems with mass ratios 1.5-8 and predict the GSF correction to the periastron advance for a nonspinning particle moving on a circular equatorial orbit around a Kerr black hole of mass $M$ and spin $S=-0.5 M^{2}$. These results are summarized in Fig. 11 below.

This paper is organized as follows. Section II explains how the periastron advance is extracted from NR simulations of binary black holes, and how the error estimates are computed. In Sec. III we establish the 3.5PN-accurate expression of the periastron advance for quasicircular orbits, including all spin-orbit and spin-spin effects. The perturbative result for a point mass orbiting a Kerr black hole on a circular equatorial orbit is obtained in Sec. IV. In Sec. V we impose known symmetries on the perturbative result and make use of this expression as a background to extract GSF information by using NR results in Sec. VI. We summarize our main findings and discuss future prospects in Sec. VII. Throughout this paper we set $G=c=1$.

\section{NUMERICAL RELATIVITY}

In this section we provide an in-depth discussion of the techniques used in Ref. [32] to extract the periastron advance from $\mathrm{BBH}$ simulations and further refine these techniques. Henceforth, we use the sum $m=m_{1}+m_{2}$ of the irreducible masses of the black holes to define dimensionless frequencies.

\section{A. Basic procedure}

The analysis of the periastron advance is based on the coordinate trajectories of the centers of the apparent horizons, as computed during BBH evolutions [34,36,43-45] using the Spectral Einstein Code (SpEC) [46]. Let $\mathbf{c}_{i}(t)$ denote the coordinates of the center of each black hole and define their relative separation $\mathbf{r}(t)=\mathbf{c}_{1}(t)-\mathbf{c}_{2}(t)$. The instantaneous orbital frequency $\Omega(t)$ is computed by

$$
\Omega(t) \equiv \frac{|\mathbf{r}(t) \times \dot{\mathbf{r}}(t)|}{r^{2}(t)},
$$

where the Euclidean cross product and norm are used, and an overdot stands for $\mathrm{d} / \mathrm{d} t$. The orbital frequency $\Omega(t)$ is the sum of a secular quasicircular piece [given by the average frequency $\Omega_{\varphi}(t)$ ] and a small oscillatory remainder containing information about the eccentricity and the radial frequency. Both components drift slowly in time due to the radiation-reaction driven inspiral of the black holes. To separate $\Omega(t)$ into these two components, we perform a fit to the model

$$
\begin{aligned}
\Omega(t)= & p_{0}\left[p_{1}-(t-T)\right]^{p_{2}} \\
& +p_{3} \cos \left[p_{4}+p_{5}(t-T)+p_{6}(t-T)^{2}\right] .
\end{aligned}
$$

The $p_{i}$ 's are parameters to be determined by the fit. The first term in Eq. (2), with fitting parameters $\left(p_{0}, p_{1}, p_{2}\right)$, is intended to capture the monotonic, nonoscillatory inspiral behavior of a noneccentric binary. Writing this as a single power-law term ensures monotonic behavior that would not be guaranteed if this term were a polynomial of order 2 or higher. The second term is designed to capture oscillations in $\Omega(t)$ that arise from orbital eccentricity. The amplitude $p_{3}$ will be proportional to the eccentricity. Because $\Omega$ is linked to the radius through angular momentum conservation, the phase of the oscillations (parameters $p_{4}, p_{5}, p_{6}$ ) will give the phase of the radial motion of the binary.

The model (2) is fitted over an interval $t \in[T-$ $\left.\frac{\Delta T}{2}, T+\frac{\Delta T}{2}\right]$ centered around the time $T$, with width $\Delta T=$ $\varpi \times 2 \pi / \Omega(T)$ parametrized by the number $\varpi$ of orbits within this interval. The instantaneous orbital frequency $\Omega_{\varphi}(T)$ and the radial frequency $\Omega_{r}(T)$ at time $T$ are computed by evaluating the monotonic and oscillatory parts of the fit at $t=T$ :

$$
\begin{aligned}
& \Omega_{\varphi}(T)=p_{0} p_{1}^{p_{2}}, \\
& \Omega_{r}(T)=p_{5} .
\end{aligned}
$$

Finally, the periastron advance is given by the ratio

$$
K_{\mathrm{NR}}(T)=\frac{\Omega_{\varphi}(T)}{\Omega_{r}(T)} .
$$

Repeating this procedure for many different times $T$ results in the periastron advance $K_{\mathrm{NR}}\left(\Omega_{\varphi}\right)$ as a function of the average quasicircular orbital frequency $\Omega_{\varphi}$. 


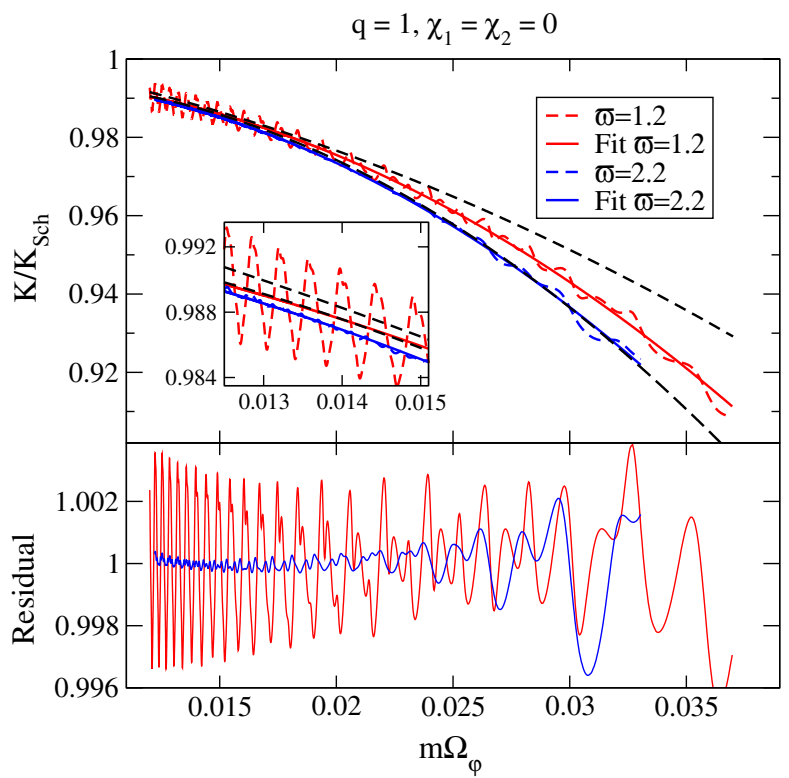

FIG. 1 (color online). Periastron advance extracted from numerical simulations. Upper panel: The dashed curves show $K_{\mathrm{NR}}\left(\Omega_{\varphi}\right) / K_{\mathrm{Sch}}\left(\Omega_{\varphi}\right)$ as computed from Eqs. (3) and (4) using fitting intervals with two different widths $\varpi$. The solid lines show polynomial fits to $K_{\mathrm{NR}} / K_{\mathrm{Sch}}$. Lower panel: Residuals of the polynomial fits.

Figure 1 shows an example of this procedure, applied to an equal-mass, nonspinning BBH system. The red-dashed and blue-dashed curves are the output of Eq. (4) for two different values of $\varpi$, normalized by the periastron advance $K_{\text {Sch }}=\left[1-6\left(m \Omega_{\varphi}\right)^{2 / 3}\right]^{-1 / 2}$ of a test mass orbiting a Schwarzschild black hole (cf. Sec. IV below) to reduce the dynamical range; note that the $y$ scale of Fig. 1 represents only a relative variation of $8 \%$ of $K_{\mathrm{NR}}$. The solid lines represent power-law fits to the dashed data, with error regions indicated by the dashed black lines. This is the procedure that was used in the analysis in Le Tiec et al. [32].

\section{B. Systematic effects}

The procedure just outlined is subject to three effects that impact $K_{\mathrm{NR}}$ at the $0.1 \%-1 \%$ level. The first of these effects is already clearly visible in Fig. $1: K_{\mathrm{NR}}\left(\Omega_{\varphi}\right)$ as obtained by Eq. (4) oscillates around its mean. These oscillations arise because the fitting function (2) does not perfectly capture the features of $\Omega(t)$ : eccentricity-related effects and the radiation-reaction driven inspiral are more complicated than the rather simple fitting formula (2) used. Early in the inspiral, these oscillations are typically of order $0.1 \%-0.2 \%$, and they grow during the inspiral. The amplitude of these oscillations is furthermore strongly dependent on the width $\varpi$ of the fitting window. This dependence arises because a longer fitting interval includes a larger number of the eccentricity-induced oscillations in $\Omega(t)$ that the fitting function (2) is designed to capture, and therefore reduces the uncertainty of the fit.

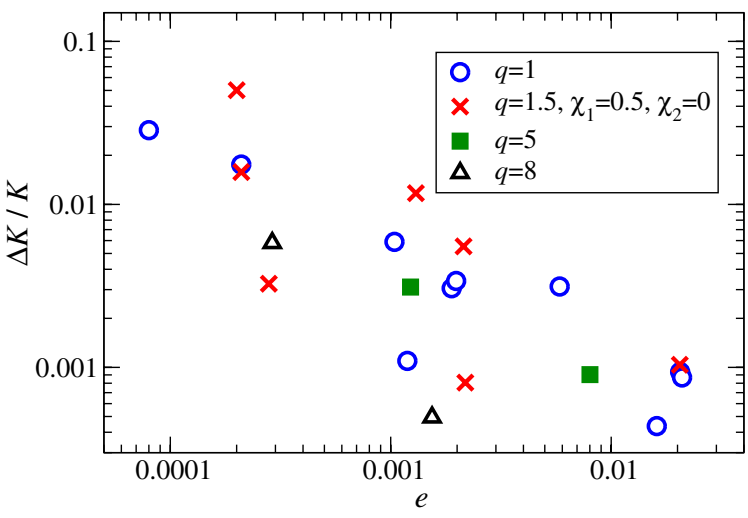

FIG. 2 (color online). Relative uncertainty $\Delta K / K$ in the numerical-relativity periastron advance as a function of the eccentricity $e$ of the configuration. Shown are data for four black-hole binaries with different mass ratios $q=m_{1} / m_{2}$, one of them with a nonzero spin. Each symbol represents a separate numerical binary black hole evolution. The results shown here were computed at the orbital frequency $m \Omega_{\varphi}=0.033$.

A second important effect enters through the magnitude of the eccentricity. The oscillatory term in Eq. (2) will be proportional to the eccentricity of the orbit. With decreasing eccentricity, this oscillatory term will be increasingly hard to isolate and $\Omega_{r}$ will be increasingly difficult to measure. This effect is illustrated in Fig. 2, which provides a survey of NR simulations at different eccentricities. An eccentricity $e \sim 0.01$ typically allows one to measure $K$ with a relative accuracy of order $0.1 \%$. For smaller eccentricities, the uncertainty in $K_{\mathrm{NR}}$ increases roughly inversely proportionally to $e$. For larger eccentricities, eventually the eccentricity-dependent corrections to the periastron advance will become noticeable; the leading relative correction is proportional to $e^{2}$, and hence still negligible for $e \sim 0.01$. Figure 2 shows data obtained at the orbital frequency $m \Omega_{\varphi}=0.033$. As one moves closer to the merger, the uncertainty $\Delta K$ increases.

A third systematic effect arises from the choice of the width $\varpi$ of the fitting interval. Larger $\varpi$ systematically underestimate $K_{\mathrm{NR}}$ because the average radial frequency over the fitting interval is biased toward larger values, as already visible in Fig. 1. Figure 3 demonstrates this drift more clearly. As can be seen, $K_{\mathrm{NR}}$ drifts by an amount of order $0.1 \%$ to $1 \%$; the drift is generally smaller at large separations (where the inspiral motion is very "small"), and more pronounced at small separations. This systematic error also gets smaller as the mass ratio of the binary increases (more unequal masses).

\section{Refined procedure}

The three effects described in Sec. II B depend strongly on the eccentricity $e$ of the run being analyzed, on the width $\varpi$ of the fitting interval, and on the orbital frequency $\Omega_{\varphi}$ under consideration for each binary configuration. All three effects couple nonlinearly and have a large impact on 


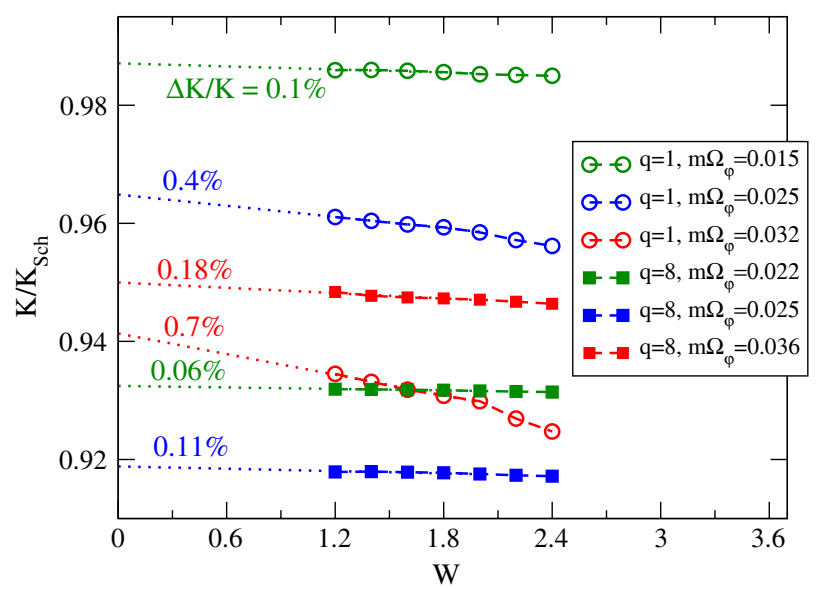

FIG. 3 (color online). Effect of the choice of width $\varpi$ on the measured periastron advance. Shown are data for the three reference frequencies $\Omega_{e / m / l}$ and for two exemplary runs: $\left(q, \chi_{1}, \chi_{2}\right)=(1,0,0)$ and $\left(q, \chi_{1}, \chi_{2}\right)=(8,0.5,0)$. The symbols denote $K_{\mathrm{NR}} / K_{\mathrm{Sch}}$ as measured with width $\varpi$ indicated on the $x$ axis. The dotted lines denote fits indicating the extrapolation to zero width, $\varpi \rightarrow 0$. The number next to each dotted line indicates the fractional change in $K_{\mathrm{NR}} / K_{\mathrm{Sch}}$ between $\varpi=1.2$ and $\varpi \rightarrow 0$. For ease of plotting, the data for $q=8$ and $m \Omega_{\varphi}=$ 0.036 has been shifted up by 0.1 .

how accurately $K_{\mathrm{NR}}$ can be measured at a given combination of $\left(e, \varpi, \Omega_{\varphi}\right)$. Furthermore, we generally do not have control over the eccentricity $e$. Numerical-relativity simulations are computationally costly. To maximize the scientific returns of these simulations, we extract the periastron advance from simulations originally performed for other purposes, even if the eccentricity is smaller than desired for optimal extraction of $K_{\mathrm{NR}}$. (The data shown in Fig. 2, based on Ref. [36], is exceptional, as the goal of these simulations was precisely the study of eccentricity.) Therefore, we proceed as follows for each BBH configuration (specified by mass ratio and spins):

(1) Pick three tentative target frequencies $\Omega_{e}, \Omega_{m}$, and $\Omega_{l}$. These are chosen to fall into the early inspiral, into the middle of the inspiral, and late in the inspiral, but such that for all three frequencies we can still obtain good periastron advance measurements.

(2) If simulations with different orbital eccentricities are available for the considered configuration, perform fits similar to those shown in Fig. 1 for each available eccentricity. Manually assess which eccentricity gives the most reliable fits (these can be different runs at the various frequencies $\left.\Omega_{e / m / l}\right)$. Determine an error bar on $K_{\mathrm{NR}}$ from manual inspection.

(3) Consider the dependence on $\varpi$ by using plots similar to Fig. 3. Take the periastron advance extrapolated to $\varpi \rightarrow 0$ as the final value reported. If the change in $K_{\mathrm{NR}}$ between $\varpi=1.2$ and $\varpi \rightarrow 0$ is larger than the error bar determined in step 2, then increase the error bar to this difference.
(4) To obtain convenient analytical approximations of the behavior of $K_{\mathrm{NR}} / K_{\mathrm{Sch}}$, fit the values for $K_{\mathrm{NR}} / K_{\mathrm{Sch}}$ at the three frequencies $\Omega_{e / m / l}$ with a quadratic polynomial in $m \Omega_{\varphi}$,

$$
\frac{K_{\mathrm{NR}}}{K_{\mathrm{Sch}}}=a_{0}+a_{1}\left(m \Omega_{\varphi}\right)+a_{2}\left(m \Omega_{\varphi}\right)^{2} .
$$

Because of the variety of simulations to be analyzed, manual inspection as indicated in the procedure above was crucial to improve the accuracy of $K_{\mathrm{NR}}$ over the earlier, more automatic procedure used in Ref. [32]. Table I lists the numerical results for the periastron advance obtained for the simulations considered here.

\section{POST-NEWTONIAN APPROXIMATION}

\section{A. Post-Newtonian calculation to 3.5PN order}

In the context of the post-Newtonian approximation to general relativity, we consider a binary system of spinning point particles (modeling two rotating black holes) with constant masses $m_{i}(i=1,2)$ and canonical spins $\mathbf{S}_{i}=$ $S_{i} \hat{\mathbf{L}}$ aligned or antialigned with the orbital angular momentum $\mathbf{L}=L \hat{\mathbf{L}}$, with $\hat{\mathbf{L}}$ the unit vector pointing in the direction of $\mathbf{L}$, such that $L>0$ and $\left|S_{i}\right|<m_{i}^{2}$. In this section, using the results of Ref. [12] we explicitly write down the PN expression of the periastron advance for circular orbits, including all spin-independent, spin-orbit (SO), and spin-spin (SS) contributions up to 3.5PN order included. Higher-order interactions in the spins $[47,48]$ will be neglected; hence we do not include the leadingorder 3.5PN terms cubic in the spins. We restrict to the conservative part of the dynamics, neglecting the dissipative effects related to gravitational-wave emission.

Reference [12] provides an explicit, 3.5PN-accurate solution of the orbital equations of motion of a binary system of spinning point particles (at quadratic order in the spins $S_{i}$ ), for a generic bound orbit and aligned or antialigned spins, in the form of a quasi-Keplerian parametrization of the motion. ${ }^{1}$ The orbital elements are expressed in terms of the two constants of the motion: the reduced binding energy $\varepsilon \equiv|E| /(m \nu)$ (recall that $E<0$ for bound orbits) and the dimensionless angular momentum $h \equiv L /\left(m^{2} \nu\right)$, where $m=m_{1}+m_{2}$ is the total mass and $\nu=m_{1} m_{2} / m^{2}$ the symmetric mass ratio, such that $\nu=1 / 4$ for equal masses and $\nu \rightarrow 0$ in the extreme mass-ratio limit. The 3.5PN expression of the (reduced) periastron advance per radial period, $K \equiv \Phi /(2 \pi)$, reads $^{2}$

\footnotetext{
${ }^{1}$ The expressions for the mean motion $n$ and periastron advance per radial period $\Phi$ as functions of $|E|$ and $L$ were not given in Ref. [12]. We thank M. Tessmer and J. Hartung for making these results available to us.

${ }^{2}$ We use the black-hole value $C_{Q}=1$ for the constant parameter characterizing the quadrupolar deformation of a compact object under the effect of its intrinsic rotation [12].
} 
TABLE I. Fitting parameters for the NR data. Here $q=m_{1} / m_{2}$ is the mass ratio, $m=m_{1}+m_{2}$ the total mass, $\chi_{i}=S_{i} / m_{i}^{2}($ with $i=1,2)$ the dimensionless spins, and $e$ the eccentricity. The fits are of the form $K=\left[a_{0}+a_{1}\left(m \Omega_{\varphi}\right)+a_{2}\left(m \Omega_{\varphi}\right)^{2}\right] /[1-$ $\left.6\left(m \Omega_{\varphi}\right)^{2 / 3}\right]^{1 / 2}$. The estimated uncertainties $K \pm \Delta K$ have a similar format. The fitting parameters $\left(a_{0}, a_{1}, a_{2}\right)$ are computed for the restricted frequency range $\Omega_{i} \leq \Omega_{\varphi} \leq \Omega_{f}$.

\begin{tabular}{|c|c|c|c|c|c|c|c|c|c|c|c|c|c|c|}
\hline \multirow[b]{2}{*}{$q$} & \multirow[b]{2}{*}{$\chi_{1}$} & \multirow[b]{2}{*}{$\chi_{2}$} & \multirow[b]{2}{*}{$10^{4} e$} & \multicolumn{3}{|c|}{$K$} & \multicolumn{3}{|c|}{$K+\Delta K$} & \multicolumn{3}{|c|}{$K-\Delta K$} & \multirow[b]{2}{*}{$m \Omega_{i}$} & \multirow[b]{2}{*}{$m \Omega_{f}$} \\
\hline & & & & $a_{0}$ & $a_{1}$ & $a_{2}$ & $a_{0}$ & $a_{1}$ & $a_{2}$ & $a_{0}$ & $a_{1}$ & $a_{2}$ & & \\
\hline 1 & 0.97 & 0.97 & 6 & 1.00764 & -3.9948 & -70.807 & 1.0065 & -3.9405 & -67.121 & 0.99417 & -2.7579 & -101.543 & 0.0169 & 0.0344 \\
\hline 1 & 0.95 & 0.95 & 1 & 0.98829 & -2.2363 & -107.11 & 0.99952 & -3.2597 & -79.724 & 0.98340 & -1.7802 & -122.724 & 0.0184 & 0.0318 \\
\hline 1 & 0.9 & 0.9 & 5 & 0.96487 & -0.3254 & -138.67 & 0.96828 & -0.5883 & -130.568 & 0.99319 & -2.6814 & -94.833 & 0.020 & 0.031 \\
\hline 1 & 0.8 & 0.8 & 5 & 0.98881 & -1.8427 & -104.636 & 1.00304 & -3.1415 & -73.025 & 0.97868 & -0.9218 & -127.882 & 0.0177 & 0.0317 \\
\hline 1 & 0.6 & 0.6 & 4 & 0.99922 & -2.0355 & -86.060 & 1.01226 & -3.1796 & -56.734 & 0.97886 & -0.2337 & -128.612 & 0.019 & 0.031 \\
\hline 1 & -0.9 & -0.9 & 7 & 0.96721 & 6.4391 & -34.411 & 1.3842 & -38.2326 & 1175.23 & 0.68088 & 37.9372 & -908.291 & 0.0177 & 0.024 \\
\hline 1 & -0.95 & -0.95 & 10 & 1.09949 & -7.4342 & 346.477 & 1.32874 & -33.6076 & 1099.42 & 0.78659 & 26.3466 & -570.337 & 0.0177 & 0.026 \\
\hline 1 & 0.5 & 0 & 3 & 0.98950 & 0.2892 & -106.77 & 1.01884 & -3.0265 & -8.075 & 0.957 & 3.8257 & -210.184 & 0.0155 & 0.025 \\
\hline 1 & 0 & 0 & 282 & 0.99554 & 0.5048 & -76.340 & 0.99678 & 0.2800 & -62.419 & 0.99430 & 0.7296 & -90.261 & 0.012 & 0.032 \\
\hline 1 & -0.5 & 0 & 4 & 0.93781 & 6.5574 & -171.793 & 1.2331 & -23.1674 & 588.235 & 0.84533 & 17.1947 & -486.223 & 0.0195 & 0.0259 \\
\hline 1.5 & 0.5 & 0 & 0.6 & 0.97522 & 1.4334 & -139.448 & 1.03313 & -4.6662 & 30.686 & 0.92706 & 6.5006 & -281.776 & 0.0158 & 0.0259 \\
\hline 1.5 & 0 & 0 & 228 & 0.99849 & 0.1745 & -66.444 & 1.00508 & -0.6835 & -36.986 & 0.99190 & 1.0326 & -95.902 & 0.013 & 0.032 \\
\hline 1.5 & -0.5 & 0 & 25 & 0.99987 & 1.0477 & -30.021 & 1.00286 & 0.6444 & -15.295 & 0.99588 & 1.5908 & -49.195 & 0.0123 & 0.0215 \\
\hline 3 & 0.5 & 0 & 3 & 1.00301 & -1.7335 & -65.616 & 1.02202 & -3.7817 & -7.465 & 0.99159 & -0.4448 & -105.151 & 0.0164 & 0.0287 \\
\hline 3 & 0 & 0 & 21 & 1.00277 & -0.0865 & -50.201 & 1.0178 & -1.5553 & -11.582 & 0.98773 & 1.3822 & -88.819 & 0.019 & 0.029 \\
\hline 3 & -0.5 & 0 & 229 & 1.00559 & 0.7584 & 17.064 & 1.01162 & 0.0920 & 38.352 & 0.99854 & 1.5502 & -8.129 & 0.013 & 0.027 \\
\hline 5 & 0.5 & 0 & 356 & 0.99812 & -1.2904 & -76.358 & 0.99779 & -1.1426 & -79.708 & 0.99845 & -1.4382 & -73.008 & 0.0169 & 0.0280 \\
\hline 5 & 0 & 0 & 367 & 0.99279 & 0.7364 & -54.033 & 1.00428 & -0.1182 & -36.789 & 0.98130 & 1.5911 & -71.276 & 0.020 & 0.041 \\
\hline 5 & -0.5 & 0 & 229 & 1.02734 & -1.3157 & 101.025 & 1.03345 & -1.9244 & 117.851 & 1.02648 & -1.2086 & 95.785 & 0.0179 & 0.036 \\
\hline 8 & 0.5 & 0 & 37 & 0.97198 & 0.7118 & -114.923 & 0.98182 & 0.0285 & -102.411 & 0.96137 & 1.4528 & -128.537 & 0.021 & 0.042 \\
\hline 8 & 0 & 0 & 84 & 0.99868 & 0.2793 & 35.300 & 1.0045 & -0.2028 & -24.723 & 0.98878 & 1.0538 & 50.982 & 0.021 & 0.036 \\
\hline 8 & -0.5 & 0 & 17 & 1.02556 & -1.2577 & 130.85 & 1.05938 & -4.3455 & 203.072 & 0.99952 & 1.2217 & 69.698 & 0.020 & 0.030 \\
\hline
\end{tabular}

$$
\begin{aligned}
K= & +\frac{3}{h^{2}}-(2+2 \Delta-\nu) \frac{\chi_{1}}{h^{3}}+\left(-\frac{15}{2}+3 \nu\right) \frac{\varepsilon}{h^{2}}+\left(\frac{105}{4}-\frac{15}{2} \nu\right) \frac{1}{h^{4}}+\left(\frac{3}{4}+\frac{3}{4} \Delta-\frac{3}{2} \nu\right) \frac{\chi_{1}^{2}}{h^{4}} \\
& +\left(12+12 \Delta-16 \nu-4 \Delta \nu+2 \nu^{2}\right) \chi_{1} \frac{\varepsilon}{h^{3}}+\left(-42-42 \Delta+\frac{147}{4} \nu+\frac{21}{4} \Delta \nu-\frac{3}{2} \nu^{2}\right) \frac{\chi_{1}}{h^{5}} \\
& +\left(\frac{1155}{4}-\left[\frac{625}{2}-\frac{615}{128} \pi^{2}\right] \nu+\frac{105}{8} \nu^{2}\right) \frac{1}{h^{6}}+\left(-\frac{315}{2}+\left[218-\frac{123}{64} \pi^{2}\right] \nu-\frac{45}{2} \nu^{2}\right) \frac{\varepsilon}{h^{4}}+\left(\frac{15}{4}-\frac{15}{4} \nu+3 \nu^{2}\right) \frac{\varepsilon^{2}}{h^{2}} \\
& +\left(\frac{105}{2}+\frac{105}{2} \Delta-135 \nu-30 \Delta \nu+\frac{45}{4} \nu^{2}\right) \frac{\chi_{1}^{2}}{h^{6}}+\left(-\frac{33}{2}-\frac{33}{2} \Delta+\frac{93}{2} \nu+\frac{27}{2} \Delta \nu-\frac{15}{2} \nu^{2}\right) \frac{\varepsilon \chi_{1}^{2}}{h^{4}} \\
& +\left(-\frac{1485}{2}-\frac{1485}{2} \Delta+\frac{15165}{16} \nu+\frac{5265}{16} \Delta \nu-\frac{345}{2} \nu^{2}-\frac{75}{8} \Delta \nu^{2}+\frac{15}{8} \nu^{3}\right) \frac{\chi_{1}}{h^{7}} \\
& +\left(420+420 \Delta-717 \nu-297 \Delta \nu+207 \nu^{2}+21 \Delta \nu^{2}-6 \nu^{3}\right) \frac{\chi_{1} \varepsilon}{h^{5}} \\
& +\left(-15-15 \Delta+42 \nu+\frac{39}{2} \Delta \nu-27 \nu^{2}-6 \Delta \nu^{2}+3 \nu^{3}\right) \frac{\chi_{1} \varepsilon^{2}}{h^{3}}+1 \leftrightarrow 2+\mathcal{O}\left(c^{-8}\right)
\end{aligned}
$$

where $\Delta \equiv\left(m_{1}-m_{2}\right) / m=\sqrt{1-4 \nu}$ is the reduced mass difference and $\chi_{1} \equiv S_{1} / m_{1}^{2}$ the dimensionless spin of particle 1 . (We assume, without any loss of generality, that $m_{1} \geq m_{2}$.) The symbol $1 \leftrightarrow 2$ stands for all the spindependent terms with the particle labels 1 and 2 exchanged $\left(\chi_{1} \leftrightarrow \chi_{2}\right.$ and $\left.\Delta \rightarrow-\Delta\right)$ that have to be added to the previous expression.
We now restrict to a circular orbit with constant azimuthal frequency $\Omega_{\varphi}$ and make use of the well-known expressions of $\varepsilon$ and $h$ as functions of the usual dimensionless, invariant PN parameter $x \equiv\left(m \Omega_{\varphi}\right)^{2 / 3}$. When including the leadingorder 1.5PN and next-to-leading order 2.5PN spin-orbit couplings, as well as the leading-order 2PN spin-spin couplings, those expressions read $[9,23,49,50]$ 


$$
\begin{aligned}
\varepsilon= & \frac{x}{2}\left\{1+\left(-\frac{3}{4}-\frac{\nu}{12}\right) x+\left(\frac{4}{3}+\frac{4}{3} \Delta-\frac{2}{3} \nu\right) \chi_{1} x^{3 / 2}+\left(-\frac{27}{8}+\frac{19}{8} \nu-\frac{\nu^{2}}{24}\right) x^{2}-\frac{1}{2}(1+\Delta-2 \nu) \chi_{1}^{2} x^{2}-\nu \chi_{1} \chi_{2} x^{2}\right. \\
& \left.+\left(4+4 \Delta-\frac{121}{18} \nu-\frac{31}{18} \Delta \nu+\frac{\nu^{2}}{9}\right) \chi_{1} x^{5 / 2}+1 \leftrightarrow 2+\mathcal{O}\left(x^{3}\right)\right\}, \\
h= & \frac{1}{\sqrt{x}}\left\{1+\left(\frac{3}{2}+\frac{\nu}{6}\right) x+\left(-\frac{5}{3}-\frac{5}{3} \Delta+\frac{5}{6} \nu\right) \chi_{1} x^{3 / 2}+\left(\frac{27}{8}-\frac{19}{8} \nu+\frac{\nu^{2}}{24}\right) x^{2}+\left(\frac{1}{2}+\frac{\Delta}{2}-\nu\right) \chi_{1}^{2} x^{2}+\nu \chi_{1} \chi_{2} x^{2}\right. \\
& \left.+\left(-\frac{7}{2}-\frac{7}{2} \Delta+\frac{847}{144} \nu+\frac{217}{144} \Delta \nu-\frac{7}{72} \nu^{2}\right) \chi_{1} x^{5 / 2}+1 \leftrightarrow 2+\mathcal{O}\left(x^{3}\right)\right\} .
\end{aligned}
$$

Note that to control the expansion for $K(x)$ up to 3.5PN order, we only need the expressions for $\varepsilon(x)$ and $h(x)$ at the relative 2.5PN accuracy. The expressions (7) can also be recovered from the quasi-Keplerian parametrization of Ref. [12], by imposing the zero-eccentricity condition $e_{t}=0$ (or equivalently $e_{r}=0$ or $e_{\varphi}=0$ ) appropriate for a circular orbit.

Replacing the formulas (7) into Eq. (6), and expanding in powers of $1 / c$, we obtain the 3.5PN result for the invariant relation $K\left(x ; \nu, \chi_{1}, \chi_{2}\right)$, which can conveniently be split into nonspinning, spin-orbit, and spin-spin contributions,

$$
K=K_{\mathrm{NS}}+K_{\mathrm{SO}}^{\mathrm{LO}}+K_{\mathrm{SS}}^{\mathrm{LO}}+K_{\mathrm{SO}}^{\mathrm{NLO}}+K_{\mathrm{SS}}^{\mathrm{NLO}}+K_{\mathrm{SO}}^{\mathrm{NNLO}}+\mathcal{O}\left(c^{-8}\right) .
$$

The nonspinning (NS) contribution $K_{\mathrm{NS}}$ is accurate to 3.5PN order. The leading-order (LO), next-to-leading order (NLO), and next-to-next-to-leading order (NNLO) SO terms $K_{\mathrm{SO}}^{\mathrm{LO}}, K_{\mathrm{SO}}^{\mathrm{NLO}}$, and $K_{\mathrm{SO}}^{\mathrm{NNLO}}$ contribute at 1.5PN, 2.5PN, and 3.5PN order, respectively. The leading-order 2PN and next-to-leading order 3PN SS contributions can themselves be split into self-spin $\left(S_{1}^{2}\right.$ and $\left.S_{2}^{2}\right)$ and cross-spin $\left(S_{1} S_{2}\right)$ interactions: $K_{\mathrm{SS}}^{\mathrm{LO}}=K_{S^{2}}^{\mathrm{LO}}+K_{S_{1} S_{2}}^{\mathrm{LO}}$ and $K_{\mathrm{SS}}^{\mathrm{NLO}}=K_{S^{2}}^{\mathrm{NLO}}+K_{S_{1} S_{2}}^{\mathrm{NLO}}$. All these contributions explicitly read

$$
\begin{aligned}
K_{\mathrm{NS}} & =1+3 x+\left(\frac{27}{2}-7 \nu\right) x^{2}+\left(\frac{135}{2}-\left[\frac{649}{4}-\frac{123}{32} \pi^{2}\right] \nu+7 \nu^{2}\right) x^{3}, \\
K_{\mathrm{SO}}^{\mathrm{LO}} & =(-2-2 \Delta+\nu) \chi_{1} x^{3 / 2}+1 \leftrightarrow 2, \\
K_{S^{2}}^{\mathrm{LO}} & =\left(\frac{3}{4}+\frac{3}{4} \Delta-\frac{3}{2} \nu\right) \chi_{1}^{2} x^{2}+1 \leftrightarrow 2, \\
K_{S_{1} S_{2}}^{\mathrm{LO}} & =3 \nu \chi_{1} \chi_{2} x^{2}, \\
K_{\mathrm{SO}}^{\mathrm{NLO}} & =\left(-17-17 \Delta+\frac{81}{4} \nu+\frac{17}{4} \Delta \nu-\nu^{2}\right) \chi_{1} x^{5 / 2}+1 \leftrightarrow 2, \\
K_{S^{2}}^{\mathrm{NLO}} & =\left(\frac{67}{4}+\frac{67}{4} \Delta-\frac{189}{4} \nu-\frac{55}{4} \Delta \nu+6 \nu^{2}\right) \chi_{1}^{2} x^{3}+1 \leftrightarrow 2, \\
K_{S_{1} S_{2}}^{\mathrm{NLO}} & =(45+2 \nu) \nu \chi_{1} \chi_{2} x^{3}, \\
K_{\mathrm{SO}}^{\mathrm{NNLO}} & =\left(-126-126 \Delta+\frac{11581}{48} \nu+\frac{5317}{48} \Delta \nu-\frac{733}{12} \nu^{2}-\frac{11}{3} \Delta \nu^{2}+\frac{\nu^{3}}{3}\right) \chi_{1} x^{7 / 2}+1 \leftrightarrow 2 .
\end{aligned}
$$

The NS contribution (9a) is a strictly increasing function of frequency for all mass ratios $(0 \leq \nu \leq 1 / 4)$. The $2 \mathrm{PN}$ and $3 \mathrm{PN} S_{1}^{2}$ and $S_{2}^{2}$ contributions (9c) and (9f) are positive for all spins and mass ratios, while the $S_{1} S_{2}$ contributions (9d) and $(9 \mathrm{~g})$ are positive if $\operatorname{sgn}\left(S_{1} S_{2}\right)>0$ and negative otherwise. The 1.5PN, 2.5PN, and 3.5PN SO contributions (9b), (9e), and (9h) are all negative (positive) when both spins are aligned (antialigned) with the angular momentum.

To ease the comparison with the perturbative result derived in Sec. IV below, we also compute the quantity $W \equiv 1 / K^{2}$ introduced in Refs. [26,51]. The 3.5PN-accurate expression for $W\left(x ; \nu, \chi_{1}, \chi_{2}\right)$ is 


$$
\begin{aligned}
W= & 1-6 x+\left[(4+4 \Delta-2 \nu) \chi_{1}+(4-4 \Delta-2 \nu) \chi_{2}\right] x^{3 / 2} \\
& +\left[14 \nu+\left(-\frac{3}{2}-\frac{3}{2} \Delta+3 \nu\right) \chi_{1}^{2}-6 \nu \chi_{1} \chi_{2}+\left(-\frac{3}{2}+\frac{3}{2} \Delta+3 \nu\right) \chi_{2}^{2}\right] x^{2} \\
& -\left[\left(2+2 \Delta+\frac{45}{2} \nu+\frac{17}{2} \Delta \nu-2 \nu^{2}\right) \chi_{1}+\left(2-2 \Delta+\frac{45}{2} \nu-\frac{17}{2} \Delta \nu-2 \nu^{2}\right) \chi_{2}\right] x^{5 / 2} \\
& +\left[\left(\frac{397}{2}-\frac{123}{16} \pi^{2}\right) \nu-14 \nu^{2}+\left(4+4 \Delta+\frac{15}{2} \nu+\frac{31}{2} \Delta \nu-9 \nu^{2}\right) \chi_{1}^{2}+(36+2 \nu) \nu \chi_{1} \chi_{2}\right. \\
+ & \left.\left(4-4 \Delta+\frac{15}{2} \nu-\frac{31}{2} \Delta \nu-9 \nu^{2}\right) \chi_{2}^{2}\right] x^{3}-\left[\left(\frac{1465}{24}+\frac{1465}{24} \Delta-\frac{373}{6} \nu-\frac{22}{3} \Delta \nu+\frac{2}{3} \nu^{2}\right) \nu \chi_{1}\right. \\
& \left.+\left(\frac{1465}{24}-\frac{1465}{24} \Delta-\frac{373}{6} \nu+\frac{22}{3} \Delta \nu+\frac{2}{3} \nu^{2}\right) \nu \chi_{2}\right] x^{7 / 2}+\mathcal{O}\left(x^{4}\right) .
\end{aligned}
$$

Note that the 3.5PN spin-orbit terms in $W$ vanish in the testparticle limit $\nu \rightarrow 0$. Recall, however, that we are missing some contributions $\mathcal{O}\left(S^{3}\right)$ at $3.5 \mathrm{PN}$ order, which may not vanish in that limit. Notice also that Eq. (10) is invariant by exchange $1 \leftrightarrow 2$ of the bodies' labels.

\section{B. Comparison to numerical-relativity simulations}

We now compare the PN predictions (8) and (9) with the NR results discussed in Sec. II. In Fig. 4 we show the fractional difference between the NR and PN predictions for $K$ as a function of spin, at different PN orders, for equal-mass black-hole binaries. We compute the periastron advance at the orbital frequency $m \Omega_{\varphi}=0.021$, which is typically in the middle of the NR frequency range. We indicate with a dot the simulations in which both black holes are spinning and with a square the simulations in which only one black hole is spinning. For spins antialigned with the orbital angular momentum, the various contributions (9) are all positive, such that the successive PN approximations approach the NR results in a monotonic way. For

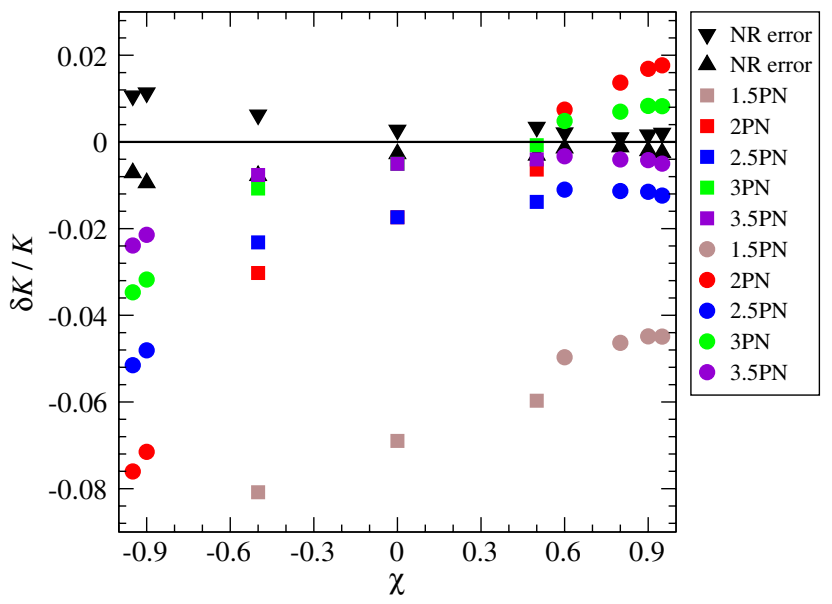

FIG. 4 (color online). Fractional difference between the NR and PN predictions for the periastron advance $K$ as a function of spin, at different PN orders, for equal-mass black-hole binaries. We set $m \Omega_{\varphi}=0.021$. spins aligned with the orbital angular momentum, the spin-squared contributions are still positive, but the spinorbit ones are negative, such that the successive PN approximations approach the NR results in a nonmonotonic way. At the moderate orbital frequency $m \Omega_{\varphi}=0.021$, the 3.5PN results are almost within the numerical errors, with a relative difference of $1 \%$ at most (except for large negative spins).

In Fig. 5 we plot the fractional difference between the $\mathrm{NR}$ and 3.5PN predictions for the periastron advance $K$, for black-hole binaries with mass ratios $q \in\{1,1.5,3,5,8\}$ and spins $\chi_{1} \in\{-0.5,0,0.5\}$ and $\chi_{2}=0$, still at the orbital frequency $m \Omega_{\varphi}=0.021$. The performance of the PN approximation deteriorates as the mass ratio increases (more unequal masses), consistent with previous findings $[32,52]$. This result is robust to changes in the orbital frequency.

Figure 6 shows the periastron advance $K$ as a function of the orbital frequency $m \Omega_{\varphi}$ for equal-mass binaries with equal spins $\chi_{1}=\chi_{2}=0.9$ (top) and $\chi_{1}=\chi_{2}=-0.9$ (bottom). We show the NR results (black continuous curves) with their errors (black dashed curves) and the PN results at different PN orders. In particular, we plot

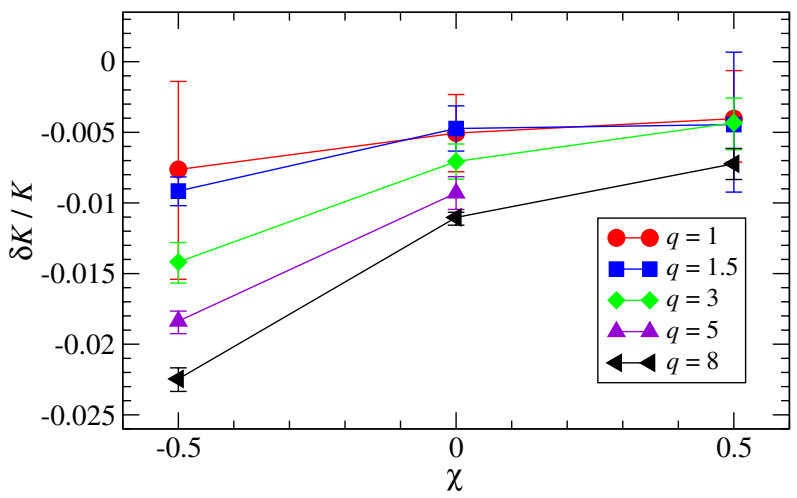

FIG. 5 (color online). Fractional difference between the NR and PN predictions for $K$ for black-hole binaries with mass ratios $q \in\{1,1.5,3,5,8\}$ and spins $\chi \equiv \chi_{1} \in\{-0.5,0,0.5\}$ and $\chi_{2}=0$. We set $m \Omega_{\varphi}=0.021$. 

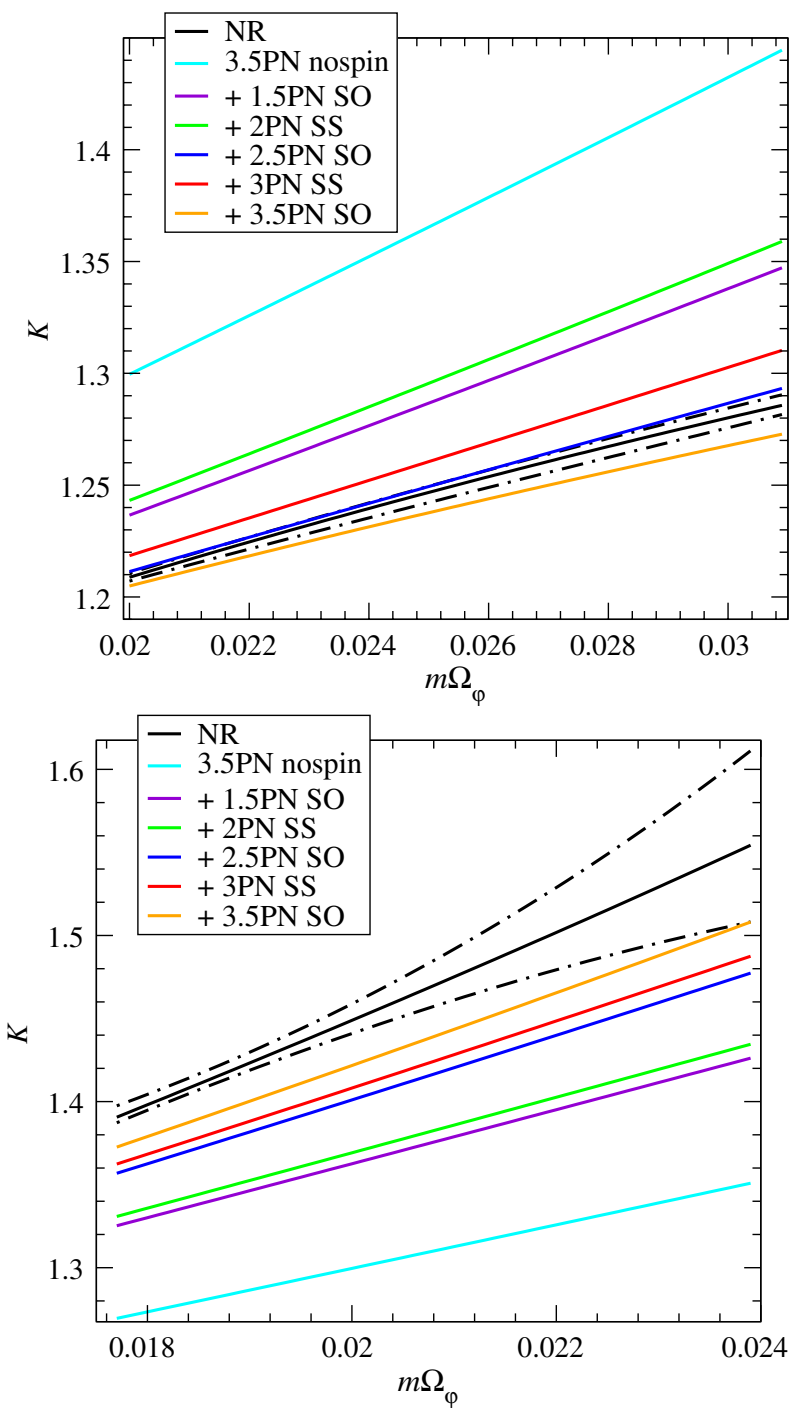

FIG. 6 (color online). Periastron advance $K$ as a function of the orbital frequency $m \Omega_{\varphi}$, for equal-mass binaries with equal spins $\chi_{1}=\chi_{2}=0.9$ (top) and $\chi_{1}=\chi_{2}=-0.9$ (bottom). The black dashed lines show the estimated numerical-relativity uncertainties.

the nonspinning 3.5PN result and show how the periastron advance varies when PN spin effects are successively added. The SO terms typically give larger contributions than the SS terms. Figure 12 shows $K$ as a function of $m \Omega_{\varphi}$ for other equal-mass, equal-spins configurations. In all cases the 3.5PN approximation underestimates the exact result, typically by a few percent over our frequency ranges.

\section{TEST-PARTICLE APPROXIMATION}

\section{A. Test mass in a Kerr background}

In this section we compute the periastron advance of a test particle on a circular orbit in the equatorial plane of a Kerr black hole; see also Refs. [19,20,53] for alternative derivations. Our analysis closely follows that of Ref. [26], in which the circular-orbit limit of the periastron advance was recently computed within the (nonspinning) EOB framework. Although the properties of timelike geodesics of the Kerr geometry were explored in detail long ago [54], we recall some well-known formulas here for the sake of completeness, in order to make our perturbative analysis self-contained.

We consider a test particle of mass $\mu$ on a bound geodesic orbit in the equatorial plane of a Kerr black hole of mass $M$ and spin $S \equiv M a \equiv M^{2} \chi$. We use Boyer-Lindquist coordinates $\{t, r, \theta, \phi\}$, defined such that the equatorial plane coincides with the plane $\theta=\pi / 2$. Using the proper time $\tau$ to parametrize the timelike geodesic followed by the particle, the orbital motion obeys

$$
\begin{aligned}
\left(\frac{\mathrm{d} r}{\mathrm{~d} \tau}\right)^{2}= & \left(e^{2}-1\right)+\frac{2 M}{r}-\frac{1}{r^{2}}\left[j^{2}+a^{2}\left(1-e^{2}\right)\right] \\
& +\frac{2 M}{r^{3}}(j-a e)^{2}, \\
r^{4}\left(\frac{\mathrm{d} \varphi}{\mathrm{d} \tau}\right)^{2}= & j-a e+a \frac{e\left(r^{2}+a^{2}\right)-a j}{r^{2}-2 M r+a^{2}}, \\
r^{4}\left(\frac{\mathrm{d} t}{\mathrm{~d} \tau}\right)^{2}= & a(j-a e)+\left(r^{2}+a^{2}\right) \frac{e\left(r^{2}+a^{2}\right)-a j}{r^{2}-2 M r+a^{2}},
\end{aligned}
$$

where $e$ and $j$ are the conserved specific energy and angular momentum of the particle. Introducing the inverse separation $u \equiv 1 / r$, and parametrizing the orbital motion in terms of the Mino time parameter $\lambda$ [55], defined such that $\mathrm{d} \tau / \mathrm{d} \lambda=r^{2}$, the radial first integral of the motion, Eq. (11a), can be rewritten in the simple form

$$
\dot{u}^{2}+V(u)=0,
$$

where the overdot stands for a derivative with respect to $\lambda$, and the radial potential $V$ is a third order polynomial in $u$,

$V=1-e^{2}-2 M u+\left[j^{2}+a^{2}\left(1-e^{2}\right)\right] u^{2}-2 M(j-a e)^{2} u^{3}$.

To derive the expression of the periastron advance in the circular-orbit limit, we can restrict to a slightly eccentric orbit, treated as a linear perturbation of an exactly circular orbit with radius $r_{0}$. To first order in a parameter $\varepsilon$ measuring the deviation from perfect circularity, the radial motion can be written as

$$
u(\lambda)=u_{0}+\varepsilon u_{1}(\lambda)+\mathcal{O}\left(\varepsilon^{2}\right),
$$

where $u_{0}=1 / r_{0}$ satisfies the circular-orbit conditions $V\left(u_{0}\right)=V^{\prime}\left(u_{0}\right)=0$. The function $u_{1}(\lambda)$ encodes the effect of the eccentricity perturbation on the radial motion. To first order in $\varepsilon$, the differential equation (12) reduces to

$$
\dot{u}_{1}^{2}+\omega_{r}^{2} u_{1}^{2}=0
$$

where $\omega_{r}^{2}\left(u_{0}\right) \equiv \frac{1}{2} V^{\prime \prime}\left(u_{0}\right)$ is the radial frequency (squared) associated with the circular orbit of radius $r_{0}$. Using the explicit expression (13) of the radial potential $V(u)$, we have 


$$
\omega_{r}^{2}=j^{2}+a^{2}\left(1-e^{2}\right)-6 M(j-a e)^{2} u_{0} .
$$

The solution of the differential equation (15) for the perturbation $u_{1}(\lambda)$ depends on the sign of the radial frequency squared: if $\omega_{r}^{2}>0$, then the perturbation is stable, as it obeys the harmonic evolution $u_{1}(\lambda) \propto \cos \left(\omega_{r} \lambda+\varphi_{0}\right)$, where $\varphi_{0}$ is a constant; if $\omega_{r}^{2}<0$, then the perturbation is unstable, as it grows like $u_{1}(\lambda) \sim \exp \left(\sqrt{-\omega_{r}^{2}} \lambda\right)$ as $\lambda \rightarrow$ $+\infty$. The boundary case $\omega_{r}^{2}=0$ corresponds to a marginally stable circular orbit, or innermost stable circular orbit (ISCO); its radius is given by

$$
r_{\mathrm{ISCO}}=\frac{6 M(j-a e)^{2}}{j^{2}+a^{2}\left(1-e^{2}\right)} .
$$

In the limit $a \rightarrow 0$ of vanishing spin, the Boyer-Lindquist radial coordinate reduces to the usual Schwarzschild radial coordinate, and we recover the well-known location $r_{\text {ISCO }}=6 M$ of the Schwarzschild ISCO.

On the other hand, the instantaneous azimuthal frequency $\omega_{\varphi} \equiv \mathrm{d} \varphi / \mathrm{d} \lambda$ of the orbit is given, in Mino time, by Eq. (11b). In the limit $\varepsilon \rightarrow 0$, it is constant and reads

$$
\omega_{\varphi}=\frac{j+2 M(a e-j) u_{0}}{1-2 M u_{0}+a^{2} u_{0}^{2}} .
$$

In the circular-orbit limit, the periastron advance is given by the ratio $K \equiv \omega_{\varphi} / \omega_{r}$ of the two frequencies of the motion. Following Refs. [26,51], we find it more convenient to work with the quantity $W \equiv 1 / K^{2}$ instead. Using Eqs. (16) and (18), we obtain

$$
\begin{aligned}
W= & {\left[j^{2}+a^{2}\left(1-e^{2}\right)-6 M(a e-j)^{2} u_{0}\right] } \\
& \times\left[\frac{1-2 M u_{0}+a^{2} u_{0}^{2}}{j+2 M(a e-j) u_{0}}\right]^{2} .
\end{aligned}
$$

Notice that the ratio of frequencies $W=\left(\omega_{r} / \omega_{\varphi}\right)^{2}$ does not depend on the time parametrization used to describe the motion; hence the result (19) is valid, e.g., in Mino time $\lambda$, in proper time $\tau$, and in Boyer-Lindquist coordinate time $t$.

Next, we use the conditions $V\left(u_{0}\right)=0$ and $V^{\prime}\left(u_{0}\right)=0$ for a circular orbit to express the energy $e$ and angular momentum $j$ as functions of the orbital radius $r_{0}$. In terms of the coordinate "velocity" $v^{2} \equiv M u_{0}=M / r_{0}$, this yields [54]

$$
\begin{aligned}
& e=\frac{1-2 v^{2}+\chi v^{3}}{\sqrt{1-3 v^{2}+2 \chi v^{3}}}, \\
& j=\frac{M}{v} \frac{1-2 \chi v^{3}+\chi^{2} v^{4}}{\sqrt{1-3 v^{2}+2 \chi v^{3}}} .
\end{aligned}
$$

Replacing these formulas into Eq. (19), the algebra simplifies considerably, and we are left with the polynomial result

$$
W=1-6 v^{2}+8 \chi v^{3}-3 \chi^{2} v^{4} .
$$

This simple expression lends itself to a nice (but simplistic) physical interpretation: the first term in the righthand side of Eq. (21) corresponds to the Newtonian result (no periastron advance), the second term encodes the full general relativistic correction for a Schwarzschild black hole ( $\chi$-independent), the third term is a spin-orbit coupling (linear in $\chi$ ), and the last term is a spin-spin contribution (quadratic in $\chi$ ).

Notice that by substituting Eqs. (20) into the expression (17) previously derived for the coordinate location of the Kerr ISCO, we obtain an equation for $v$ that can easily be shown to be equivalent to the vanishing of the polynomial in the right-hand side of Eq. (21). This is expected because the condition $W=0$ corresponds to a vanishing radial frequency (independently of the time parametrization used), which defines the ISCO $[54,56]$.

The test-particle result (21) being expressed in terms of the Boyer-Lindquist coordinate radius $r_{0}$ of the circular orbit, a meaningful comparison with the predictions from PN theory and NR simulations is not obvious. To ease such comparisons, we must first relate $r_{0}$ to the "invariant" circular-orbit frequency $\Omega_{\varphi} \equiv \mathrm{d} \varphi / \mathrm{d} t$, defined in terms of the coordinate time $t$ that coincides with the proper time of an asymptotic, inertial observer. By taking the ratio of the first integrals (11b) and (11c) for $\mathrm{d} \varphi / \mathrm{d} \tau$ and $\mathrm{d} t / \mathrm{d} \tau$, we find

$\Omega_{\varphi}=\frac{u_{0}^{2}\left[j+2 M(a e-j) u_{0}\right]}{e+a u_{0}^{2}\left[a e+2 M(a e-j) u_{0}\right]}=\left(a+\frac{M}{v^{3}}\right)^{-1}$,

where we used Eqs. (20) to substitute $e$ and $j$ in favor of $v$. Inverting this last result yields the expression of $v^{2}=M u_{0}$ in terms of the dimensionless product $M \Omega_{\varphi}$ as [54]

$$
v^{3}=\frac{M \Omega_{\varphi}}{1-\chi M \Omega_{\varphi}} .
$$

Substituting this expression into Eq. (21), we finally obtain the desired relationship $W\left(M \Omega_{\varphi} ; \chi\right)$, valid in the test-mass limit. In the limit $\chi \rightarrow 0$ of vanishing spin, the result (21) reduces to the well-known expression $W=1-6\left(M \Omega_{\varphi}\right)^{2 / 3}$ for the periastron advance of a test particle on a circular orbit around a Schwarzschild black hole $[9,18]$.

A check of the validity of (21) is provided by the results of Schmidt [19], who performed a thorough analysis of the fundamental frequencies of the geodesic motion of a test particle on a generic (bound) orbit around a Kerr black hole. Combining Eqs. (40)-(42), (51), and (59)-(62) of Ref. [19] with Eqs. (20) of this paper, the result (21) can easily be recovered. That expression was also established in Sec. 2.5 of Ref. [53].

\section{B. Test spin in a Kerr background}

Before ending this section, we consider the additional effects on the periastron advance $W$ if the particle has a spin. Using a pole-dipole-quadrupole model (gravitational 
skeleton approach) for the small black hole, the authors of the companion paper [20] computed the periastron advance for a spinning particle of mass $\mu$ and spin $S_{*} \equiv \mu^{2} \chi_{*}$ orbiting a Kerr black hole of mass $M$ and spin $S=M^{2} \chi$, for circular equatorial orbits and spins aligned or antialigned with the orbital angular momentum. Thereafter, it will prove convenient to introduce the notation $\bar{q} \equiv 1 / q$ for the inverse mass ratio, such that $0<\bar{q} \leq 1$ and the perturbative limit corresponds to $\bar{q} \rightarrow 0$. Discarding the terms quadratic in the spin variable $\bar{\chi}_{*} \equiv \bar{q} \chi_{*}$, the authors of Ref. [20] found

$$
\begin{aligned}
W= & 1-6 v^{2}+\left(8 \chi+6 \bar{\chi}_{*}\right) v^{3}-\left(3 \chi^{2}+6 \chi \bar{\chi}_{*}\right) v^{4} \\
& -18 \bar{\chi}_{*} v^{5}+30 \chi \bar{\chi}_{*} v^{6}-12 \chi^{2} \bar{\chi}_{*} v^{7}+\mathcal{O}\left(\chi_{*}^{2}\right) .
\end{aligned}
$$

Even when accounting for the terms linear in the spin $S_{*}$ of the small black hole, the result for the coordinate-invariant function $W\left(M \Omega_{\varphi} ; \chi, \chi_{*}\right)$ takes the form of a polynomial in the "velocity" $v^{2}=M / r_{0}$, given by Eq. (23) above. Note that higher powers in the spins appear at increasingly higher PN orders: 1.5PN, 2PN, and 3.5PN for linear (spin-orbit), quadratic (spin-spin), and cubic contributions. Since $0 \leq|\chi|,\left|\chi_{*}\right|<1$, contributions of high order in the spins are further suppressed when $v \leq 1$.

To make contact with the PN result (10), valid for any mass ratio, we substitute (23) in the expression (24), and expand the result in powers of the dimensionless PN parameter $y \equiv\left(M \Omega_{\varphi}\right)^{2 / 3}$ in the weak-field/small-velocity limit $M \Omega_{\varphi} \rightarrow 0$. At 3.5PN order, we obtain

$$
\begin{aligned}
W= & 1-6 y+\left(8 \chi+6 \bar{q} \chi_{*}\right) y^{3 / 2}-\left(3 \chi^{2}+6 \bar{q} \chi_{*} \chi\right) y^{2} \\
& -\left(4 \chi+18 \bar{q} \chi_{*}\right) y^{5 / 2}+\left(8 \chi^{2}+36 \bar{q} \chi_{* \chi} \chi y^{3}\right. \\
& -\left(4 \chi^{3}+20 \bar{q} \chi_{*} \chi^{2}\right) y^{7 / 2}+\mathcal{O}\left(y^{4}, \chi_{*}^{2}\right) .
\end{aligned}
$$

This expression is in complete agreement with the testmass limit ( $\nu \rightarrow 0$ and $\Delta \rightarrow 1$ ) of the PN result (10), as long as the mass $M$ and spin $\chi$ of the Kerr black hole, and the mass $\mu$ and spin $\chi_{*}$ of the particle, are identified with $\left(m_{1}, \chi_{1}\right)$ and $\left(m_{2}, \chi_{2}\right)$, respectively. In that limit the symmetric mass ratio reduces to $\nu=\bar{q}+\mathcal{O}\left(\bar{q}^{2}\right)$. Note that we would need to control the (unknown) contribution $\mathcal{O}\left(S^{3}\right)$ at 3.5PN order in the PN result to compare with the term $\mathcal{O}\left(y^{7 / 2}\right)$ in Eq. (25).

\section{IMPOSING A KNOWN SYMMETRY ON THE PERTURBATIVE RESULT}

\section{A. Motivation and guidance from post-Newtonian theory}

In the general relativistic two-body problem, most quantities of physical interest are symmetric by exchange of the bodies' labels. For compact-object binaries on quasicircular orbits, this property is satisfied, e.g., by the periastron advance, the binding energy, the total angular momentum, the fluxes of energy and angular momentum, and the gravitational-wave polarizations themselves, when expressed as functions of the circular-orbit frequency. This symmetry property can be seen in explicit PN expansions for these relations, such as Eq. (10) above, Eqs. (3.13) and (3.15) of Ref. [57], or Eqs. (194), (231), and (237)-(241) of Ref. [7]. In the context of black hole perturbation theory, however, the central Kerr black hole and the small spinning compact object are, by design, not treated "on equal footing." Any quantity of interest is usually computed as an expansion in powers of the usual mass ratio $\bar{q}=\mu / M$, and is therefore not symmetric by exchange of the black hole and the particle.

One could hardly overstate the major role played by symmetries in physics. Symmetry considerations often drastically simplify the process of solving a given physics problem. References [58,59] provide an example of the constraining power of symmetries in the context of the binary black-hole problem in general relativity. In the present context, enforcing the symmetry by exchange $1 \leftrightarrow 2$ on the perturbative expression (24) could possibly enlarge the domain of validity of this relativistic formula. However, starting from Eq. (24), one can devise many ways of imposing this symmetry property. We shall look for the simplest such "symmetrization," guided solely by well-established properties of the PN expansion.

Let us consider two spinning particles with masses $m_{i}$ and spins $S_{i}=m_{i}^{2} \chi_{i}$, on a quasicircular orbit with azimuthal frequency $\Omega_{\varphi}$. The PN expansion of any function $f$ that is symmetric under the exchange $1 \leftrightarrow 2$ of the particles' labels, and scales like $(v / c)^{0}$ at Newtonian order, takes the generic form ${ }^{3}$

$$
\begin{aligned}
f\left(\Omega_{\varphi} ;\right. & \left.m_{i}, S_{i}\right) \\
= & \sum_{n=0}^{N} a_{n}(\nu) x^{n / 2}+x^{3 / 2} \sum_{n=0}^{N-3}\left[b_{n}(\nu) \chi_{s}+c_{n}(\nu) \Delta \chi_{a}\right] x^{n / 2} \\
& +x^{2} \sum_{n=0}^{N-4}\left[d_{n}(\nu) \chi_{s}^{2}+e_{n}(\nu) \chi_{s} \Delta \chi_{a}+f_{n}(\nu) \chi_{a}^{2}\right] x^{n / 2} \\
& +x^{7 / 2} \sum_{n=0}^{N-7}\left[g_{n}(\nu) \chi_{s}^{3}+h_{n}(\nu) \chi_{s}^{2} \Delta_{\chi_{a}}\right. \\
& \left.+i_{n}(\nu) \chi_{s} \chi_{a}^{2}+j_{n}(\nu) \Delta \chi_{a}^{3}\right] x^{n / 2}+o\left(x^{N / 2}\right)
\end{aligned}
$$

with $N \geq 7$ a fixed integer. The coefficients $a_{n}, b_{n}, c_{n}, \ldots$, are polynomials in the symmetric mass ratio $\nu$, and we introduced the half-sum and half-difference of the dimensionless spins,

\footnotetext{
${ }^{3}$ Because of gravitational tail effects, a logarithmic running appears starting at the relative 4PN order [60]. See, e.g., Ref. [61] and references therein. We neglect those here to simplify the discussion.
} 


$$
\begin{aligned}
& \chi_{s} \equiv \frac{1}{2}\left(\chi_{1}+\chi_{2}\right), \\
& \chi_{a} \equiv \frac{1}{2}\left(\chi_{1}-\chi_{2}\right) .
\end{aligned}
$$

Note that $\Delta \rightarrow-\Delta$ by exchange $1 \leftrightarrow 2$ of the particles' labels, such that the product $\Delta \chi_{a}$ appearing in Eq. (26) is indeed symmetric. There is, of course, no unique way to write down the dependence on the spins $\chi_{1}$ and $\chi_{2}$ in the PN expansion (26). However, given the present emphasis on symmetries, the variables $\chi_{s}$ and $\chi_{a}$ (or rather $\Delta \chi_{a}$ ) provide a natural choice, as Eq. (10) above suggests.

\section{B. Substitution rules for masses and spins}

While the perturbative result (24), or rather its PN expansion (25), is most easily expressed in terms of the variables $\left(y, \bar{q}, \chi, \chi_{*}\right)$, the generic PN formula (26) features the variables $\left(x, \nu, \chi_{s}, \chi_{a}\right)$. Therefore, to impose the symmetry by exchange $1 \leftrightarrow 2$ on the perturbative result (24), the mass $M$ of the Kerr black hole should be replaced by the sum $m=m_{1}+m_{2}$ of the component masses, and the asymmetric mass ratio $\bar{q}$ by the symmetic mass ratio $\nu$ :

$$
\begin{aligned}
& y=\left(M \Omega_{\varphi}\right)^{2 / 3} \rightarrow x=\left(m \Omega_{\varphi}\right)^{2 / 3}, \\
& \bar{q}=\mu / M \rightarrow \nu=m_{1} m_{2} / m^{2} .
\end{aligned}
$$

The substitution (28a) is commonly used while comparing results from perturbative calculations to those of numerical relativity simulations, the post-Newtonian approximation, or the EOB model $[26,31,32,40,51,62]$. As was pointed out earlier, the symmetric mass ratio $\nu=\bar{q} /(1+\bar{q})^{2}$ appears most naturally in PN calculations, and for small mass ratios we have $\nu=\bar{q}+\mathcal{O}\left(\bar{q}^{2}\right)$, or equivalently $\bar{q}=\nu+\mathcal{O}\left(\nu^{2}\right)$. These considerations motivated Refs. [32,37,40,41] to adopt the substitution (28b) while comparing the results of perturbative calculations to those of NR simulations.

Next, we note that in the test-mass limit $\nu \rightarrow 0$ the spin $\chi_{2}$ of the lightest body must disappear from Eq. (26), which can only depend on $m_{2} \Omega_{\varphi}=M \Omega_{\varphi}$ and $\chi_{1}=\chi$ in that limit; recall e.g. Eq. (21) with (23). This implies that the polynomials $b_{n}(\nu), c_{n}(\nu), d_{n}(\nu), f_{n}(\nu), g_{n}(\nu), j_{n}(\nu), \ldots$, in Eq. (26) must satisfy $b_{n}(0)=c_{n}(0), d_{n}(0)=f_{n}(0), g_{n}(0)=$ $j_{n}(0)$, etc. This motivates substituting the spin $\chi$ of the Kerr black hole in Eq. (24) by the following symmetric linear combination of the spin variables $\chi_{s}$ and $\chi_{a}$ :

$$
\chi \rightarrow \chi_{0} \equiv \chi_{s}+\Delta \chi_{a} .
$$

This replacement will indeed ensure that all terms $\mathcal{O}\left(\nu^{0}\right)$, including the terms $\mathcal{O}\left(\Delta \nu^{0}\right)$, will be reproduced by the PN expansion of the symmetric version of the perturbative formula (24). An immediate consequence of the substitutions (28a) and (29) is the following replacement:

$$
v^{2}=\frac{y}{\left(1-\chi y^{3 / 2}\right)^{2 / 3}} \rightarrow u^{2} \equiv \frac{x}{\left(1-\chi_{0} x^{3 / 2}\right)^{2 / 3}} .
$$

Comparing the PN expansion (25) of the formula (24), valid in the test-particle limit, with the generic PN expansion (26), valid for any mass ratio, it is clear that the numerical coefficients in front of the terms $\mathcal{O}\left(\bar{q} \chi_{*}\right)$ in (25) come from the sum of the numerical coefficients in front of the terms $\mathcal{O}\left(\nu \chi_{2}\right)$ and $\mathcal{O}\left(\Delta \nu \chi_{2}\right)$ in Eq. (10), as $\Delta \rightarrow 1$ when $\nu \rightarrow 0$. Hence, following the substitution (29) of $\chi$ by a linear combination of $\chi_{s}$ and $\Delta \chi_{a}$, we make the following substitution for the spin $\chi_{*}$ of the small body:

$$
\chi_{*} \rightarrow c_{s} \chi_{s}+c_{a}\left(\Delta \chi_{a}\right),
$$

where $c_{s}$ and $c_{a}$ are a priori unknown coefficients. The spin $\chi_{*}$ occurs at five different places in (24), each time multiplying a different power of the velocity $v$. Importantly, the coefficients $c_{s}$ and $c_{a}$ need not take the same numerical values in each of these five terms, contrary to the unique substitution (29) for $\chi$.

Finally, we point out that one could add in Eqs. (29) or (31) any symmetric function of the masses and spins that vanish in the limit $\nu \rightarrow 0$. We refrain from doing so, making only the simplest substitutions compatible with the structure of the PN expansion, since we do not have any guiding principle motivating the introduction of additional mass-ratio corrections.

\section{Symmetric background}

We now need to determine the values of the coefficients $c_{s}$ and $c_{a}$ in each of the five occurrences of $\chi_{*}$. This is done by making the substitutions (28)-(31) into Eq. (24), expanding the result in powers of $x$ up to 3.5PN order, expanding again in powers of the mass ratio $\bar{q}$ to first order, and enforcing agreement with the PN expansion (25) of the perturbative result (24). Doing so and remembering that there can be no term $\mathcal{O}(\Delta \nu)$ or $\mathcal{O}\left(\nu^{2}\right)$ in the 1.5PN SO and 2PN SS contributions, we obtain the unique solutions $\left(c_{s}, c_{a}\right)=(-2 / 3,0)$ and $\left(c_{s}, c_{a}\right)=(0,0)$ for the terms $\mathcal{O}\left(u^{3}\right)$ and $\mathcal{O}\left(u^{4}\right)$. Furthermore, we find the relationships $c_{s}=c_{a}+28$ for the term $\mathcal{O}\left(u^{5}\right), c_{s}=c_{a}+44$ for the term $\mathcal{O}\left(u^{6}\right)$, and $c_{s}=c_{a}-16$ for the term $\mathcal{O}\left(u^{7}\right)$. Our final formula for the "symmetrized" version of the perturbative result (24) thus reads

$$
\begin{aligned}
W_{\mathrm{SB}}= & 1-6 u^{2}+\left(8 \chi_{0}-4 \nu \chi_{s}\right) u^{3}-3 \chi_{0}^{2} u^{4} \\
& -\nu\left[(\alpha+28) \chi_{s}+\alpha \Delta \chi_{a}\right] u^{5} \\
& +\nu\left[(\beta+44) \chi_{s}+\beta \Delta \chi_{a}\right] \chi_{0} u^{6} \\
& +\nu\left[(\gamma-16) \chi_{s}+\gamma \Delta \chi_{a}\right] \chi_{0}^{2} u^{7} .
\end{aligned}
$$

By construction, Eq. (32) is symmetric by exchange $1 \leftrightarrow 2$ of the bodies' labels, and it reduces to the known result (24) in the extreme mass-ratio limit $\nu \ll 1$. This expression effectively encodes some spin-dependent finite mass-ratio corrections through $\nu, \Delta$, and $\chi_{0}=\chi_{s}+\Delta \chi_{a}$. Hereafter, we will refer to Eq. (32) as the symmetric background (SB), and we will use it in Sec. VI as the zeroth-order 
approximation, or background, for a new type of expansion in powers of the symmetric mass ratio $\nu$.

The numerical values of the coefficients $(\alpha, \beta, \gamma)$ are left unconstrained by our "symmetrization." However, by considering the PN expansion of Eq. (32), and using some information from the PN result (10), namely the coefficients $45 / 2$ and $15 / 2$ in front of the terms $\mathcal{O}\left(\nu x^{5 / 2}\right)$ and $\mathcal{O}\left(\nu x^{3}\right)$, we readily fix the values of two of the coefficients as

$$
\begin{aligned}
& \alpha=17, \\
& \beta=11 .
\end{aligned}
$$

Unfortunately, we would need to know the contribution $\mathcal{O}\left(S^{3}\right)$ at $3.5 \mathrm{PN}$ order in Eq. (10) to fix the value of $\gamma$.
Nevertheless, we checked that for the range of frequencies, mass ratios, and spins for which we have NR data, any value $|\gamma| \leq 100$ affects $W_{\mathrm{SB}}$ at the relative $0.2 \%$ level at most. This is because the term $\mathcal{O}\left(u^{7}\right)$ in Eq. (32) is cubic in the spins and contributes at leading 3.5PN order. Henceforth, we shall thus use (simply out of convenience) the fiducial value $\gamma_{\mathrm{fid}}=0$ in Eq. (32). A future PN calculation of the leading-order contribution $\mathcal{O}\left(S^{3}\right)$ in the periastron advance would immediately provide the unique, correct value of the coefficient $\gamma$.

Hence, in the weak-field/small velocity limit $m \Omega_{\varphi} \rightarrow 0$, the 3PN expansion of the symmetric background (32) and (33) reads

$$
\begin{aligned}
W_{\mathrm{SB}}= & 1-6 x+\left[(4+4 \Delta-2 \nu) \chi_{1}+(4-4 \Delta-2 \nu) \chi_{2}\right] x^{3 / 2} \\
& +\left[\left(-\frac{3}{2}-\frac{3}{2} \Delta+3 \nu\right) \chi_{1}^{2}-6 \nu \chi_{1} \chi_{2}+\left(-\frac{3}{2}+\frac{3}{2} \Delta+3 \nu\right) \chi_{2}^{2}\right] x^{2} \\
& -\left[\left(2+2 \Delta+\frac{45}{2} \nu+\frac{17}{2} \Delta \nu\right) \chi_{1}+\left(2-2 \Delta+\frac{45}{2} \nu-\frac{17}{2} \Delta \nu\right) \chi_{2}\right] x^{5 / 2} \\
& +\left[\left(4+4 \Delta+\frac{15}{2} \nu+\frac{31}{2} \Delta \nu-11 \nu^{2}\right) \chi_{1}^{2}+(36+22 \nu) \nu \chi_{1} \chi_{2}+\left(4-4 \Delta+\frac{15}{2} \nu-\frac{31}{2} \Delta \nu-11 \nu^{2}\right) \chi_{2}^{2}\right] x^{3}+\mathcal{O}\left(x^{7 / 2}\right) .
\end{aligned}
$$

Comparing with the PN result (10), we find that the fully relativistic, symmetric background (32) and (33) reproduces the exact leading-order 1.5PN spin-orbit and 2PN spinspin terms, which are of course valid for any mass ratio. ${ }^{4}$ It also reproduces the next-to-leading order 2.5PN spin-orbit and 3PN spin-spin terms, except for the contributions $\mathcal{O}\left(\nu^{2}\right)$; these five quadratic terms could nonetheless be encoded in $W_{\mathrm{SB}}$ by imposing the symmetry by exchange $1 \leftrightarrow 2$ to the known terms $\mathcal{O}\left(\chi_{*}^{2}\right)$ [20] in the perturbative result (24). Furthermore, because the test-spin expression (24) does not include any spin-independent mass-ratio correction [ $\bar{q}$ always appears in factors of $\chi_{*}$ in Eq. (24)], the formulas (32) and (33) cannot reproduce the masstype contributions $\mathcal{O}(\nu)$ and $\mathcal{O}\left(\nu^{2}\right)$ at $2 \mathrm{PN}$ and $3 \mathrm{PN}$ orders in Eq. (10).

\section{EXTRACTING SELF-FORCE INFORMATION FROM NUMERICAL-RELATIVITY SIMULATIONS}

Using the symmetric background (32) and (33), we introduce a new type of perturbative expansion in Sec. VI A. This allows us to use the results of NR simulations detailed

\footnotetext{
${ }^{4}$ The variable $S_{0}=4 m^{2} \chi_{0}$ was previously introduced, in a PN context, as an effective spin that fully encodes the leading-order 2PN spin-spin terms in the Hamiltonian of two spinning particles [63]. Hence it is not surprising that the substitution (29) allows one to reproduce the exact $2 \mathrm{PN}$ spin-spin terms in the periastron advance.
}

in Sec. II to measure the GSF correction to the geodesic periastron advance of a particle orbiting a Schwarzschild (Kerr) black hole in Sec. VIB (Sec. VIC). Finally, in Sec. VID we compare the predictions of the new perturbative expansion to the NR results for equal-mass, equalspin configurations.

\section{A. Expansion in the symmetric mass ratio}

In the PN approximation, one usually expands all quantities in powers of the small PN parameter $x=\left(m \Omega_{\varphi}\right)^{2 / 3}$, with coefficients depending on the symmetric mass ratio $\nu$ and the spins $\chi_{i}$ [see Eq. (26)]; these coefficients encode all finite mass-ratio corrections at each PN order. By contrast, in black-hole perturbation theory, one usually expands all quantities in powers of the small (asymmetric) mass ratio $\bar{q}$, with coefficients depending on $y=\left(M \Omega_{\varphi}\right)^{2 / 3}$ and the spin $\chi$ of the central Kerr black hole; these coefficients encode all the relativistic corrections at each perturbative order.

Motivated by the generic form (26) of the PN expansion, as well as by the earlier works [32,37-42] suggesting that the scaling $\bar{q} \rightarrow \nu=\bar{q} /(1+\bar{q})^{2}$ considerably extends the domain of validity of perturbative calculations, we introduce a new type of expansion in powers of the symmetric mass ratio, with coefficients encoding all the relativistic corrections at each order, using the symmetric background (32) and (33) as the zeroth-order approximation. Therefore, we are considering a formal expansion of the type 


$$
W=W_{\mathrm{SB}}+\sum_{n=1}^{\infty} \nu^{n} W_{n},
$$

where the functions $W_{n}\left(\Omega_{\varphi} ; m_{i}, S_{i}\right)$ encode the successive finite mass-ratio corrections to the background $W_{\mathrm{SB}}$. The symmetry by exchange of the bodies' labels implies that these functions can always be written in the form

$W_{n}\left(\Omega_{\varphi} ; m_{i}, S_{i}\right)=f_{n}\left(x, \chi_{s}, \chi_{a}^{2}\right)+\Delta \chi_{a} g_{n}\left(x, \chi_{s}, \chi_{a}^{2}\right)$,

where $f_{n}$ and $g_{n}$ are functions of the symmetric variables $x$, $\chi_{s}$, and $\chi_{a}^{2}$. The traditional PN and perturbative approximations are then recovered by expanding the formal series (35) and (36) in powers of $x$ and $\bar{q}$, respectively.

Notice that the functions $W_{n}$ implicitly depend on the mass ratio $\bar{q}$ through the reduced mass difference $\Delta=$ $\sqrt{1-4 \nu}$ appearing in front of $g_{n}$ in Eq. (36). However, from the PN expansions (10) and (34) of $W$ and $W_{\mathrm{SB}}$ we have the leading-order scalings $f_{1}=\mathcal{O}\left(x^{2}\right)$ and $g_{1}=$ $\mathcal{O}\left(x^{7 / 2}\right)$. Thus $g_{1} \ll f_{1}$ in the frequency range $0.05 \lesssim x \lesssim$ 0.1 for which we have NR data, such that $W_{1} \simeq f_{1}$ depends only weakly on the mass ratio. For nonspinning binaries, $\chi_{s}=\chi_{a}=0$, we simply have $W_{\mathrm{SB}}=1-6 x$ and $W_{n}=$ $f_{n}(x)$ is independent of the mass ratio.

\section{B. Self-force in a Schwarzschild background}

Figure 7 shows the difference $\delta W \equiv W_{\mathrm{NR}}-W_{\mathrm{SB}}$ between the NR results for $W=1 / K^{2}$ and the symmetric background, as a function of the orbital frequency $m \Omega_{\varphi}$, for nonspinning black-hole binaries with mass ratios $q \in$ $\{1,1.5,3,5,8\}$. The various differences $\delta W$ are of order 0.01-0.07, showing that the background accounts for about $90 \%$ of the exact result, for all mass ratios considered.

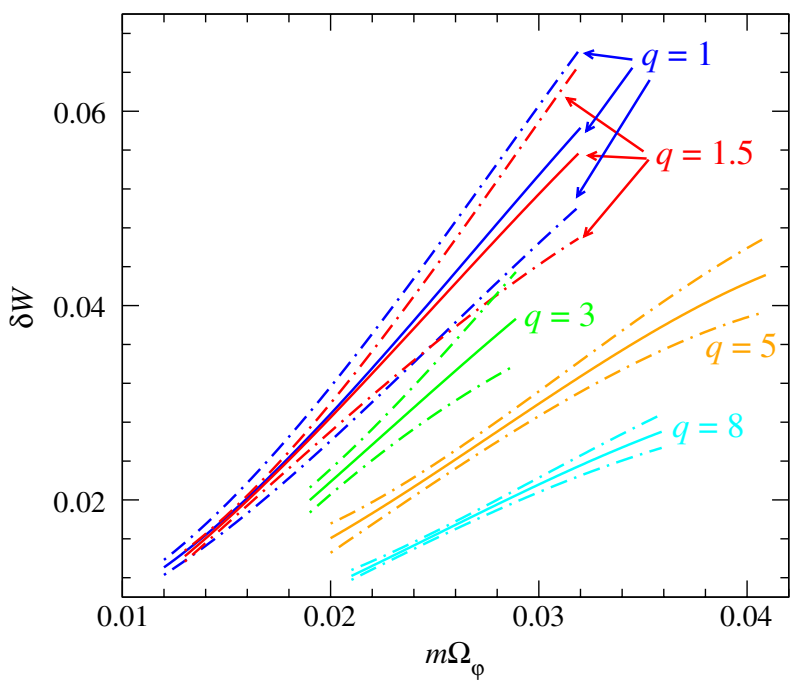

FIG. 7 (color online). The difference $\delta W=W_{\mathrm{NR}}-W_{\mathrm{SB}}$ as a function of the orbital frequency $m \Omega_{\varphi}$, for nonspinning binaries with mass ratios $q=1$ (blue lines), 1.5 (red lines), 3 (green lines), 5 (orange lines), and 8 (cyan lines). The dashed lines show the estimated NR uncertainties.
Notice that $\delta W\left(\Omega_{\varphi}\right)$ depends sensitively on the mass ratio $q$. In Fig. 8 the differences $\delta W$ are rescaled by the symmetric mass ratio $\nu$, still for mass ratios $q \in\{1,1.5,3,5,8\}$. The bottom panel shows that the five independent curves for $\delta W / \nu$ overlap very well over a wide range of orbital frequencies. Their scatter is much smaller than the intrinsic NR error bars shown in the upper panel. The remarkable alignment of the various curves for $\delta W / \nu$ implies that (i) the fully relativistic numerical results for $W$ are well approximated by an expansion of the type (35), and that (ii) the finite massratio corrections $\mathcal{O}\left(\nu^{2}\right)$ or higher are significantly smaller than the sum of the contributions $\mathcal{O}\left(\nu^{0}\right)$ and $\mathcal{O}(\nu)$. Hence, the overlapping curves in the bottom panel of Fig. 8 effectively measure the function $W_{1}\left(m \Omega_{\varphi}\right)$ appearing in Eq. (35) over the frequency range $0.012<m \Omega_{\varphi}<0.041$, which corresponds to a range of separations $8 m \leqq r_{\Omega} \lesssim 19 m$, where $r_{\Omega} \equiv\left(m / \Omega_{\varphi}^{2}\right)^{1 / 3}$. We find that the numerical data can be captured by the compact analytic formula

$$
W_{1}^{\mathrm{fit}}=14 x^{2} \frac{1+c_{1} x}{1+c_{2} x+c_{3} x^{2}},
$$

where $c_{1}, c_{2}, c_{3}$ are fitting coefficients. The formula (37) accounts for the leading-order (2PN) behavior of $W_{1}(x)$ when $x \rightarrow 0$ [see Eq. (10) above]. It was first introduced in Ref. [51] to model the GSF correction to the periastron advance of a particle orbiting a Schwarzschild black hole. We find for the best fit coefficients (the superscript stands for "nonspinning")

$$
\begin{aligned}
& c_{1}^{\mathrm{ns}}=-5.4022, \\
& c_{2}^{\mathrm{ns}}=-11.1172, \\
& c_{3}^{\mathrm{ns}}=38.8701 .
\end{aligned}
$$

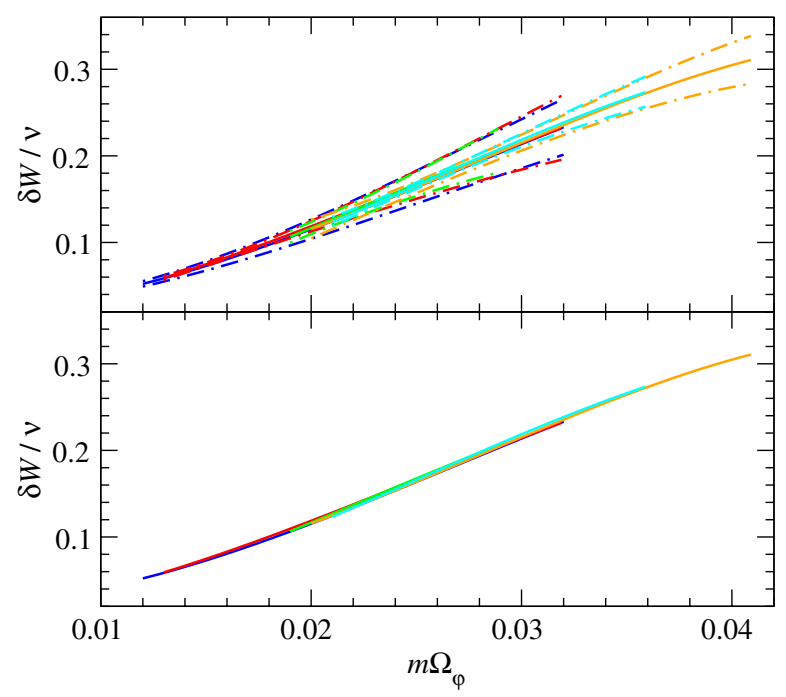

FIG. 8 (color online). The rescaled difference $\delta W / \nu$ as a function of $m \Omega_{\varphi}$, for nonspinning binaries, including (top) and excluding (bottom) the uncertainties affecting the NR results. 
As long as the dissipative radiation-reaction effects related to the emission of gravitational waves can be neglected, the first-order correction $W_{1}$ to $W_{\mathrm{SB}}$ coincides with the conservative piece of the GSF contribution to the periastron advance, say $W_{\mathrm{GSF}}$. This function was computed in Ref. [51] with high numerical accuracy. The authors performed several fits of the GSF data for $W_{\mathrm{GSF}}(x)$ in the range $6 m<r_{\Omega}<80 m$. In particular, they found that these data can be accurately reproduced at the $2.4 \times 10^{-3}$ level by means of the fitting formula (37), with best fit coeffcients $c_{1}=13.3687, c_{2}=4.60958$, and $c_{3}=-9.47696$. Figure 11 shows that the fit (37) and (38) of the NR results for $W_{\mathrm{GSF}}(x)$ closely tracks the exact perturbative result [51] (blue line) up to $m \Omega_{\varphi} \simeq 0.03$. The difference grows at larger frequencies, but remains within the NR uncertainty down to separations of order $r_{\Omega} \simeq 9 \mathrm{~m}$, while the 3.5PN prediction (red line) overshoots over the entire frequency range.

\section{Self-force in a Kerr background}

Next, we repeat the analysis of Sec. VIB in the case of spinning black-hole binaries with mass ratios $q \in$ $\{1.5,3,5,8\}$ and spins $\chi_{1}=-0.5$ and $\chi_{2}=0$. (We do not use the NR data for $q=1$ because it has much larger error bars than the other configurations; see the left panel of Fig. 5.) In Fig. 9 we plot the difference $\delta W=W_{\mathrm{NR}}-W_{\mathrm{SB}}$ for these configurations. As in the nonspinning case, the background accounts for more than $90 \%$ of the full result and $\delta W$ depends strongly on $q$.

Figure 10 shows the rescaled difference $\delta W / \nu$, still for mass ratios $q \in\{1.5,3,5,8\}$ and spins $\chi_{1}=-0.5$ and $\chi_{2}=0$. Again the mean values align remarkably well, with little scatter. As discussed earlier, in our frequency

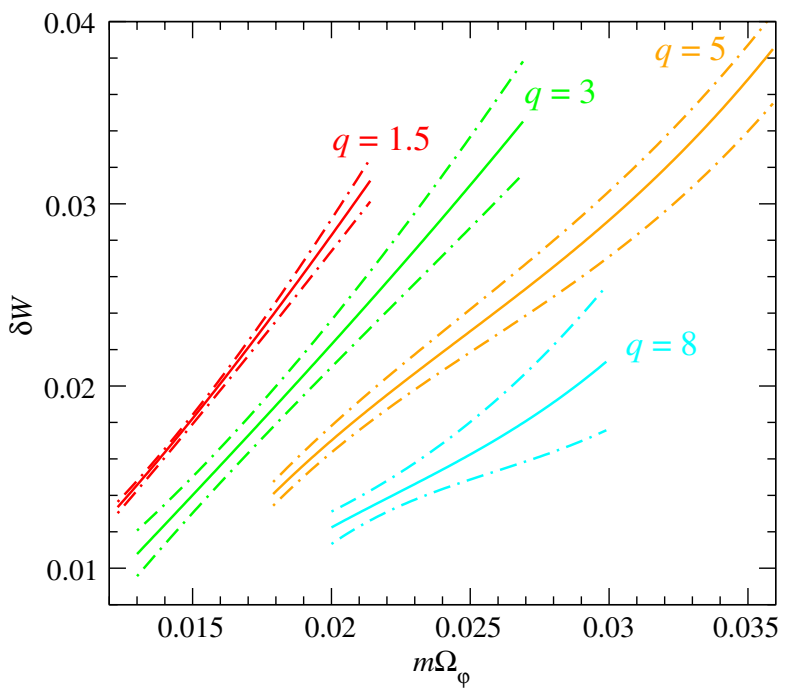

FIG. 9 (color online). The difference $\delta W=W_{\mathrm{NR}}-W_{\mathrm{SB}}$ as a function of the orbital frequency $m \Omega_{\varphi}$, for spinning binaries with $\left(\chi_{1}, \chi_{2}\right)=(-0.5,0)$ and mass ratios $q=1.5$ (red lines), 3 (green lines), 5 (orange lines), and 8 (cyan lines). The dashed lines show the estimated NR uncertainties. range the first-order correction $W_{1}$ to $W_{\mathrm{SB}}$ depends only weakly on the mass ratio $q$. The overlapping curves in the bottom panel of Fig. 10 thus measure the function $W_{1}\left(\Omega_{\varphi}\right)$ over the frequency range $0.012<m \Omega_{\varphi}<0.036$, corresponding to separations $9 m \lesssim r_{\Omega} \lesssim 19 m$. Combining the NR results for the various mass ratios and performing a least-squares fit to the model (37), we obtain the best fit values (the superscript stands for "spin down")

$$
\begin{aligned}
& c_{1}^{\text {down }}=1.1973, \\
& c_{2}^{\text {down }}=-6.88457, \\
& c_{3}^{\text {down }}=37.3406 .
\end{aligned}
$$

Interestingly, the fits (37) and (38) and (37)-(39) of the NR results for the nonspinning $\left(\chi_{1}=0\right)$ and spinning $\left(\chi_{1}=\right.$ -0.5 ) configurations agree to within $4 \%$ over their common frequency range $0.012<m \Omega_{\varphi}<0.036$. Therefore, the effects of the spin of the most massive black hole are almost entirely accounted for by the symmetric background $W_{\mathrm{SB}}$.

The prediction (37)-(39) should be compared with a future calculation of the conservative part of the GSF correction to the periastron advance of a nonspinning particle on a circular, equatorial orbit around a Kerr black hole of mass $M$ and spin $S=-0.5 M^{2}$. Given the conventions usually adopted within the self-force community, such a future perturbative calculation would likely be formulated as an expansion in powers of the mass ratio $\bar{q}=$ $\mu / M$ about a Kerr background. However, following Refs. [21,32,51] and keeping with the PN habit of using the total mass $M+\mu$ to adimensionalize frequencies (rather than the mass $M$ of the central black hole), we shall consider an expansion of the type

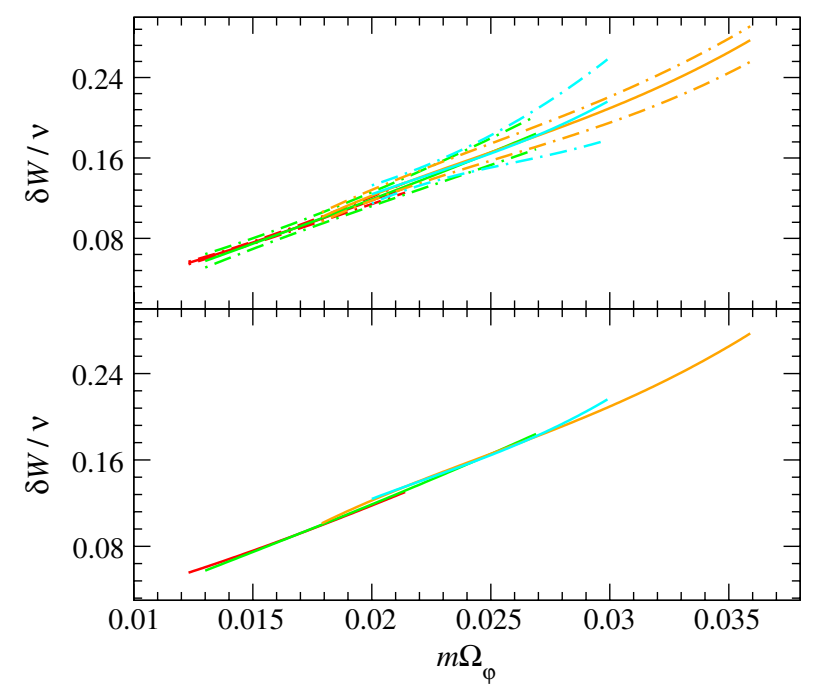

FIG. 10 (color online). The rescaled difference $\delta W / \nu$ as a function of $m \Omega_{\varphi}$, for spinning binaries with $\left(\chi_{1}, \chi_{2}\right)=$ $(-0.5,0)$, including (top) and excluding (bottom) the uncertainties affecting the NR results. 


$$
W=W_{\mathrm{Kerr}}(x ; \chi)+\bar{q} W_{\mathrm{GSF}}(x ; \chi)+\mathcal{O}\left(\bar{q}^{2}\right),
$$

where $W_{\text {Kerr }}$ is given by Eqs. (21) and (23) with $M \rightarrow M+$ $\mu$, and $x=\left[(M+\mu) \Omega_{\varphi}\right]^{2 / 3}$. The expression (40) should be compared to the expansion (35), in which the formula (32) for the symmetric background $W_{\mathrm{SB}}$ must be expanded in powers of $\bar{q}$ to first order, using the spins values $\chi_{s}=$ $\chi_{a}=\chi / 2$. Comparing the two expressions, we obtain the following relationship between the GSF correction $W_{\mathrm{GSF}}$ to the Kerr result and our first-order symmetric mass-ratio correction $W_{1}$ :

$$
\begin{aligned}
W_{\mathrm{GSF}}= & W_{1}-10 \chi v^{3}+6 \chi^{2} v^{4}-27 \chi v^{5}+25 \chi^{2} v^{6} \\
& +(\gamma-4) \chi^{3} v^{7} .
\end{aligned}
$$

Here, the "velocity" $v$ is given by Eq. (23) with $M \rightarrow$ $M+\mu$. [Recall that the numerical coefficient $\gamma$ will remain unknown until the terms $\mathcal{O}\left(S^{3}\right)$ at 3.5PN order in Eq. (10) are computed, but that its precise numerical value is irrelevant for $x \leqslant 0.12$.] The additional spin-dependent terms in Eq. (41) come from the mass-ratio expansion of the symmetric background $W_{\mathrm{SB}}$. For a Schwarzschild black hole we simply have $W_{\mathrm{GSF}}=W_{1}$; see the discussion at the end of Sec. VIB. The PN expansion of $W_{\mathrm{GSF}}-W_{1}$ recovers all the spin-dependent terms $\mathcal{O}(\bar{q})$ in Eq. (10) with $\chi_{1}=\chi$ and $\chi_{2}=0$, except for the 3.5PN term linear in $\chi$ whose effect must be captured in $W_{1}(x ; \chi)$.

For a Kerr black hole with spin $\chi=-0.5$, one should replace $W_{1}$ in Eq. (41) by the fit (37)-(39). The GSF correction (41) for $\chi=-0.5$ (with $\gamma_{\mathrm{fid}}=0$ ) is plotted in Fig. 11. Clearly, the effect of the spin of the central black hole on the rate of periastron advance is significant: the

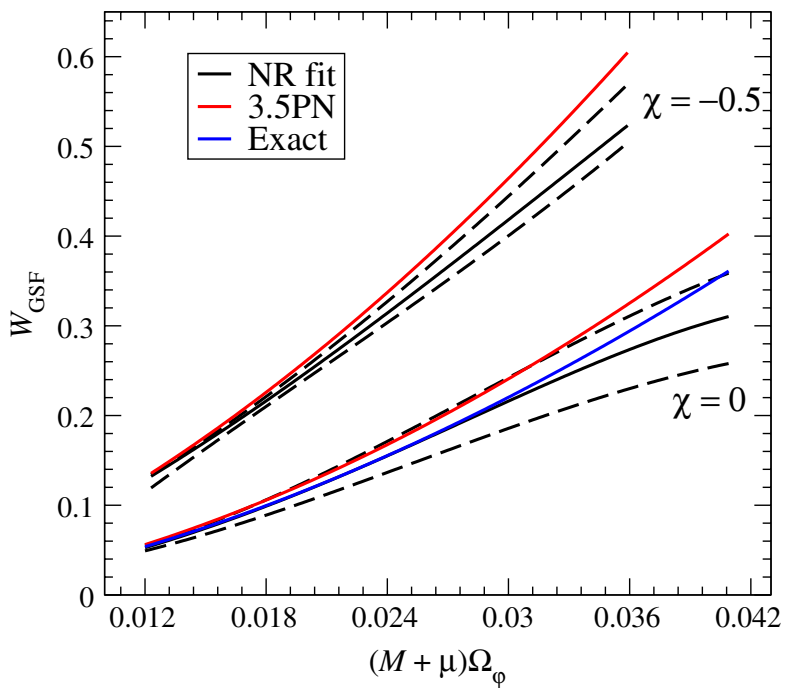

FIG. 11 (color online). Gravitational self-force correction $W_{\mathrm{GSF}}$ to the periastron advance of a nonspinning particle of mass $\mu$ orbiting a black hole of mass $M$ and spin $S \equiv \chi M^{2}$, as measured using NR simulations of black-hole binaries with mass ratios $q=1,1.5,3,5,8$. Also shown are the 3.5PN prediction (red lines) and the exact result for $\chi=0$ (blue lines).
GSF correction is more than doubled with respect to the nonspinning case. In particular we find that for retrograde orbits, the spin yields a decrease in the self-force contribution to $K=1 / \sqrt{W}$. However, given the error estimates on the NR results, our measurement of $W_{\mathrm{GSF}}$ is only accurate at the 5\%-10\% level. The 3.5PN approximation for $W_{\mathrm{GSF}}$ (red curve) clearly deviates from the NR-based prediction. It will be interesting to see how the exact GSF result compares with these predictions.

\section{Comparison for equal-mass, equal-spin configurations}

In the previous two subsections, we relied upon the input from NR simulations to measure conservative GSF effects on the periastron advance for nonspinning $\mathrm{BBH}$ and binaries with one nonzero spin. In this subsection we shall invert that logic, comparing the prediction of perturbation theory (symmetrized in the masses and spins) to those of NR simulations of equal-mass binaries with equal spins $\chi_{1}=\chi_{2}=-0.95, \quad-0.9, \quad 0,0.6,0.8,0.9, \quad 0.95,0.97$. Figure 12 shows that the predictions of the improved perturbative expansion (35) used to first order in $\nu$ (red curves), with $W_{1}$ given by the exact GSF result in a Schwarzschild background, are in very good agreement with the NR results (black curves), even for nearly extremal spins.

Importantly, the red curves in Fig. 12 were plotted using the inverse sum $\left(W_{\mathrm{SB}}+\nu W_{1}\right)^{-1 / 2}$, without any further expansion in powers of the symmetric mass ratio, because $W_{\mathrm{SB}}$ depends implicitly on $\nu$ through the spin variable $\chi_{0}=\chi_{s}+\Delta \chi_{a}$. Note also that the improved perturbative expression does not include all the correct spin information. Indeed, as pointed out in Sec. V C, the symmetric background $W_{\mathrm{SB}}$ does not capture the 3.5PN spin-orbit terms, nor the $\mathcal{O}\left(\nu^{2}\right)$ contributions to the $2.5 \mathrm{PN}$ spin-orbit and 3PN spin-spin terms. A proper comparison between the expansion (35) used to first order in $\nu$ and the NR results should make use of the (so far unknown) GSF correction to the periastron advance of a spinning particle in a Kerr background; here we merely made use of the GSF correction to the periastron advance of a nonspinning particle in a Schwarzschild background.

\section{E. Discussion of the results}

We conclude that, at least for the cases studied in this paper (see Refs. [37-42] for other examples), the expansion (35) in powers of $\nu$ used to first order provides a better approximation to the exact NR results than the usual PN expansion (10) used to third order. Loosely speaking, this observation suggests that relativistic corrections dominate over finite mass-ratio corrections. This striking observation can be understood, at a heuristic level, as follows:

(i) In the formal expansion (35), the mass-ratio corrections $W_{n}(n \geq 2)$ are suppressed by factors of $\nu^{n}$ and $\nu^{n-1}$ relative to the leading-order contributions $W_{\mathrm{SB}}$ 

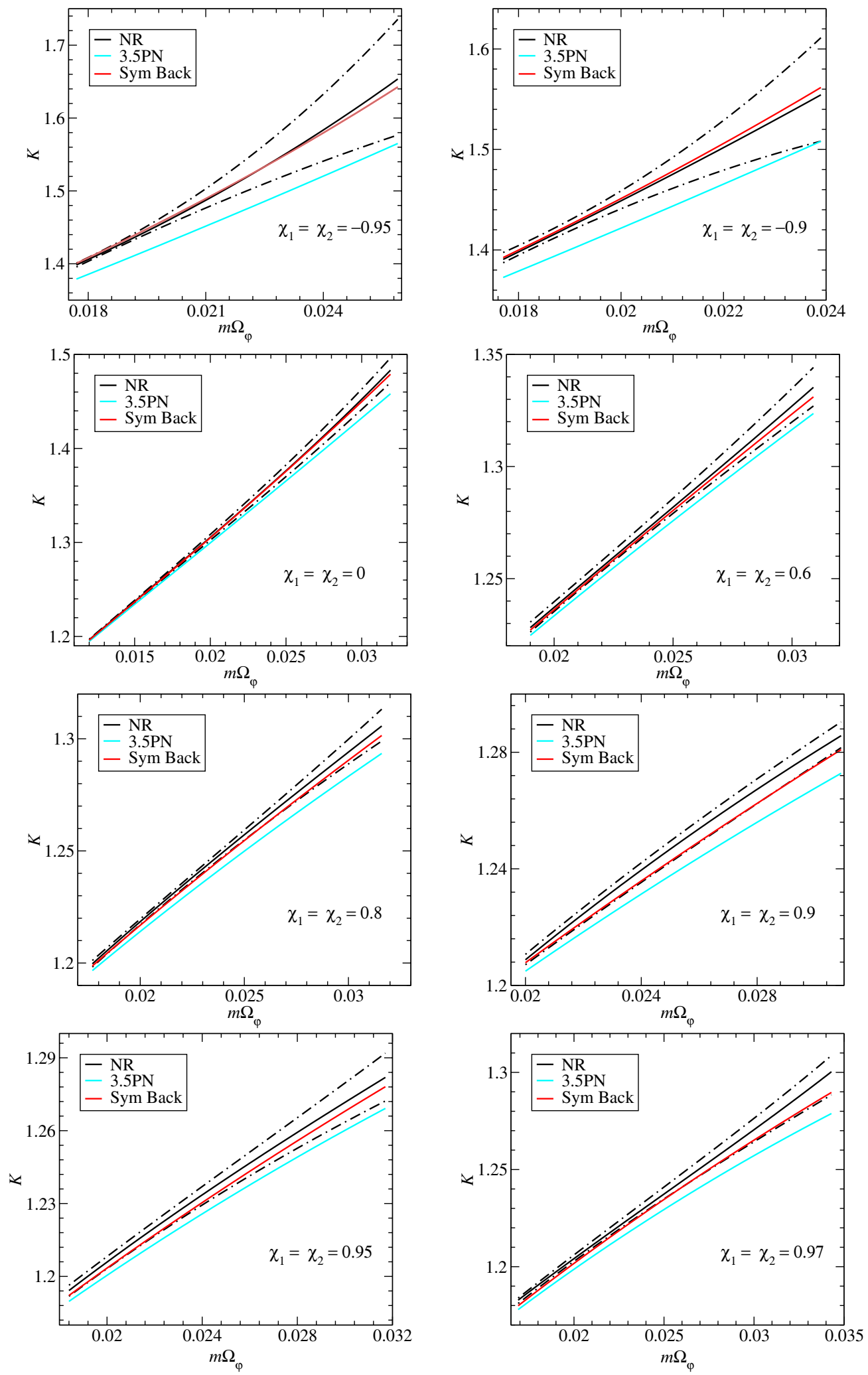

FIG. 12 (color online). The periastron advance $K$ as a function of the circular-orbit frequency $m \Omega_{\varphi}$ for equal-mass, equal-spin configurations, as computed using NR simulations (black lines), post-Newtonian theory to 3.5PN order (cyan lines), and the improved perturbative expansion (35) to first order (red lines). 
and $W_{1}$, where the symmetric mass ratio ranges in $0<\nu \leq 1 / 4$

(ii) The contribution $\mathcal{O}\left(\nu^{n}\right)$ in Eq. (35) does not appear before the $n \mathrm{PN}$ order; i.e., higher mass-ratio corrections are further suppressed by increasingly high powers of the orbital velocity $0<v \leqslant 0.3$.

For larger orbital frequencies (smaller separations), the NR results become much less accurate (see Sec. II), such that it becomes difficult to assess whether the additional corrections $\mathcal{O}\left(\nu^{2}\right)$ and higher become significant, in which case the mass-ratio degeneracy observed in Figs. 8 and 10 would be lifted. Furthermore, as the binary gets increasingly closer to the final plunge and merger, the adiabatic approximation must break down and purely conservative effects on the periastron advance can no longer be disentangled from the dissipative effects of radiation reaction. A comparison to the conservative piece of the GSF correction to the geodesic periastron advance then becomes meaningless.

\section{SUMMARY AND PROSPECTS}

We have studied the periastron advance in binary systems of spinning black holes on quasicircular orbits, for spins aligned or antialigned with the orbital angular momentum, by using NR simulations, the PN approximation, and black-hole perturbation theory. For the range of orbital frequencies, mass ratios, and spins considered, the 3.5PN approximation reproduces the NR results to within a few percent; this (dis)agreement deteriorates with increasing frequency and mass ratio (more unequal masses).

Motivated by the mathematical structure of the PN expansion, we then devised a simple method to impose the symmetry by exchange of the bodies' labels on the perturbative formula. The resulting "symmetric background" recovers most spin effects up to $3 \mathrm{PN}$ order. We then introduced a new type of expansion in powers of the symmetric mass ratio, using the symmetric background as a zeroth-order approximation. This allowed us, by comparison to the NR results, to measure the GSF correction to the periastron advance of a nonspinning particle orbiting a black hole of mass $M$ and spin $S=-0.5 M^{2}$. This is one of the first results encoding the effect of the conservative GSF on the motion of a particle in a Kerr background; see [64] for another example. That such a milestone was obtained by combining information from NR simulations, PN expansions, and black-hole perturbations illustrates the powerful interplay of these approximation methods and numerical techniques.

Numerical relativity simulations can thus be used to gain information regarding perturbative GSF effects on the dynamics of compact-object binaries. However, given the high computational cost and limited accuracy of such simulations, using NR data to develop accurate templates for extreme mass ratio inspirals is unpractical; clearly, standard perturbative methods [15-17] are far better suited to model the dynamics and gravitational-wave emission of such systems.

However, this work supports the idea that by inverting the logic followed in Secs. VI A, VI B, and VIC, the results of perturbative GSF calculations may prove useful for the development of accurate waveforms for binary systems of spinning compact objects with moderate mass ratios; see Sec. VID. The "symmetrization" introduced in Sec. V could in principle be applied to other coordinate-invariant diagnostics of the binary dynamics and wave emission, such as the binding energy, the total angular momentum, the fluxes of energy and angular momentum, and the gravitational-wave polarizations themselves. The addition of finite mass-ratio corrections coming from perturbative GSF calculations on top of such symmetric backgrounds, using perturbative expansions of the type (35), suggests a novel method to devise highly accurate approximations to the exact results, even for comparable-mass binaries.

\section{ACKNOWLEDGMENTS}

A.B. and A.L.T. acknowledge support from NSF through Grants No. PHY-0903631 and No. PHY1208881. A. B. also acknowledges support from NASA through Grant No. NNX09AI81G and A. L. T. from the Maryland Center for Fundamental Physics. A. M. and H. P. acknowledge support from NSERC of Canada, from the Canada Research Chairs Program, and from the Canadian Institute for Advanced Research. D. H., L. K., G. L., and S. T. acknowledge support from the Sherman Fairchild Foundation and NSF Grants No. PHY-1306125 and No. PHYS-1005426 at Cornell. M.S., B.S., and N.T. gratefully acknowledge support from the Sherman Fairchild Foundation and NSF Grants No. PHY-1068881, No. PHY-1005655, and No. DMS-1065438 at Caltech. The numerical relativity simulations were performed at the GPC supercomputer at the SciNet HPC Consortium [65]; SciNet is funded by the Canada Foundation for Innovation (CFI) under the auspices of Compute Canada; the Government of Ontario; Ontario Research Fund-Research Excellence; and the University of Toronto. Further computations were performed on the Caltech computer cluster Zwicky, which was funded by the Sherman Fairchild Foundation and the NSF MRI-R2 Grant No. PHY0960291, on SHC at Caltech, which is supported by the Sherman Fairchild Foundation, and on the NSF XSEDE network under Grant No. TG-PHY990007N. 
[1] A. Einstein, Sitzber. Preuss. Akad. Wiss. 47, 831 (1915).

[2] I. H. Stairs, Living Rev. Relativity 6, 5 (2003).

[3] D. R. Lorimer, Living Rev. Relativity 11, 8 (2008).

[4] D. Shoemaker (LIGO Collaboration), LIGO Document Report No. T0900288-v3, 2010.

[5] F. Acernese et al., Classical Quantum Gravity 25, 114045 (2008).

[6] eLISA, http://www.elisascience.org.

[7] L. Blanchet, Living Rev. Relativity 9, 4 (2006).

[8] H. P. Robertson, Ann. Math. 39, 101 (1938).

[9] T. Damour and G. Schäfer, Nuovo Cimento Soc. Ital. Fis. B 101, 127 (1988).

[10] T. Damour, P. Jaranowski, and G. Schäfer, Phys. Rev. D 62, 044024 (2000).

[11] M. Tessmer, J. Hartung, and G. Schäfer, Classical Quantum Gravity 27, 165005 (2010).

[12] M. Tessmer, J. Hartung, and G. Schäfer, Classical Quantum Gravity 30, 015007 (2013).

[13] C. Königsdörffer and A. Gopakumar, Phys. Rev. D 71, 024039 (2005).

[14] M. Tessmer, Phys. Rev. D 80, 124034 (2009).

[15] M. Sasaki and H. Tagoshi, Living Rev. Relativity 6, 6 (2003).

[16] L. Barack, Classical Quantum Gravity 26, 213001 (2009).

[17] E. Poisson, A. Pound, and I. Vega, Living Rev. Relativity 14, 7 (2011).

[18] C. Cutler, D. Kennefick, and E. Poisson, Phys. Rev. D 50, 3816 (1994).

[19] W. Schmidt, Classical Quantum Gravity 19, 2743 (2002).

[20] T. Hinderer et al., Phys. Rev. D 88, 084005 (2013).

[21] L. Barack and N. Sago, Phys. Rev. D 83, 084023 (2011).

[22] A. Buonanno and T. Damour, Phys. Rev. D 59, 084006 (1999).

[23] T. Damour, P. Jaranowski, and G. Schäfer, Phys. Rev. D 62, 084011 (2000).

[24] T. Damour, P. Jaranowski, and G. Schäfer, Phys. Rev. D 78, 024009 (2008).

[25] E. Barausse and A. Buonanno, Phys. Rev. D 81, 084024 (2010).

[26] T. Damour, Phys. Rev. D 81, 024017 (2010).

[27] F. Pretorius, Phys. Rev. Lett. 95, 121101 (2005).

[28] J. G. Baker, J. Centrella, D.-I. Choi, M. Koppitz, and J. van Meter, Phys. Rev. Lett. 96, 111102 (2006).

[29] M. Campanelli, C. O. Lousto, P. Marronetti, and Y. Zlochower, Phys. Rev. Lett. 96, 111101 (2006).

[30] H.P. Pfeiffer, Classical Quantum Gravity 29, 124004 (2012).

[31] A.H. Mroué, H.P. Pfeiffer, L.E. Kidder, and S. A. Teukolsky, Phys. Rev. D 82, 124016 (2010).

[32] A. Le Tiec, A. H. Mroué, L. Barack, A. Buonanno, H. P. Pfeiffer, N. Sago, and A. Taracchini, Phys. Rev. Lett. 107, 141101 (2011).

[33] F. Foucart, L. Buchman, M. D. Duez, M. Grudich, L. E. Kidder, I. MacDonald, A. Mroue, H. P. Pfeiffer, M. A. Scheel, and B. Szilagyi, Phys. Rev. D 88, 064017 (2013).
[34] D. A. Hemberger, G. Lovelace, T. J. Loredo, L. E. Kidder, M. A. Scheel, B. Szilágyi, N.W. Taylor, and S. A. Teukolsky, Phys. Rev. D 88, 064014 (2013).

[35] G. Lovelace, M. Boyle, M. A. Scheel, and B. Szilágyi, Classical Quantum Gravity 29, 045003 (2012).

[36] A. H. Mroué and H. P. Pfeiffer, arXiv:1210.2958.

[37] L. Smarr, in Sources of Gravitational Radiation, edited by L. Smarr (Cambridge University Press, Cambridge, England, 1979), p. 245.

[38] M. J. Fitchett and S. Detweiler, Mon. Not. R. Astron. Soc. 211, 933 (1984).

[39] M. Favata, S. A. Hughes, and D. E. Holz, Astrophys. J. 607, L5 (2004).

[40] U. Sperhake, V. Cardoso, C. D. Ott, E. Schnetter, and H. Witek, Phys. Rev. D 84, 084038 (2011).

[41] A. Le Tiec, E. Barausse, and A. Buonanno, Phys. Rev. Lett. 108, 131103 (2012).

[42] A. Nagar, arXiv:1306.6299.

[43] A. H. Mroué et al., arXiv:1304.6077.

[44] L. T. Buchman, H. P. Pfeiffer, M. A. Scheel, and B. Szilágyi, Phys. Rev. D 86, 084033 (2012).

[45] I. MacDonald, A. H. Mroué, H. P. Pfeiffer, M. Boyle, L. E. Kidder, M. A. Scheel, B. Szilágyi, and N. W. Taylor, Phys. Rev. D 87, 024009 (2013).

[46] SpEC, http://www.black-holes.org/SpEC.html.

[47] S. Hergt and G. Schäfer, Phys. Rev. D 77, 104001 (2008).

[48] S. Hergt and G. Schäfer, Phys. Rev. D 78, 124004 (2008).

[49] L. Blanchet, A. Buonanno, and G. Faye, Phys. Rev. D 74, 104034 (2006); 75, 049903(E) (2007); 81，089901(E) (2010).

[50] T. Damour, P. Jaranowski, and G. Schäfer, Phys. Rev. D 77, 064032 (2008).

[51] L. Barack, T. Damour, and N. Sago, Phys. Rev. D 82, 084036 (2010).

[52] E. Barausse, A. Buonanno, and A. Le Tiec, Phys. Rev. D 85, 064010 (2012).

[53] M. A. Abramowicz and P.C. Fragile, Living Rev. Relativity 16, 1 (2013).

[54] J. M. Bardeen, W.H. Press, and S.A. Teukolsky, Astrophys. J. 178, 347 (1972).

[55] Y. Mino, Phys. Rev. D 67, 084027 (2003).

[56] M. Favata, Phys. Rev. D 83, 024028 (2011).

[57] A. Bohé, S. Marsat, and L. Blanchet, Classical Quantum Gravity 30, 135009 (2013).

[58] L. Boyle, M. Kesden, and S. Nissanke, Phys. Rev. Lett. 100, 151101 (2008).

[59] L. Boyle and M. Kesden, Phys. Rev. D 78, 024017 (2008).

[60] L. Blanchet and T. Damour, Phys. Rev. D 37, 1410 (1988).

[61] L. Blanchet, S. Detweiler, A. Le Tiec, and B. F. Whiting, Phys. Rev. D 81, 084033 (2010).

[62] S. Akcay, L. Barack, T. Damour, and N. Sago, Phys. Rev. D 86, 104041 (2012).

[63] T. Damour, Phys. Rev. D 64, 124013 (2001).

[64] A. G. Shah, J. L. Friedman, and T. S. Keidl, Phys. Rev. D 86, 084059 (2012).

[65] C. Loken et al., J. Phys. Conf. Ser. 256, 012026 (2010). 